\title{
ROSINA - ROSETTA ORBITER SPECTROMETER FOR ION AND NEUTRAL ANALYSIS
}

\author{
H. BALSIGER ${ }^{1}$, K. ALTWEGG ${ }^{1, *}$, P. BOCHSLER ${ }^{1}$, P. EBERHARDT ${ }^{1}$, J. FISCHER ${ }^{1}$, \\ S. GRAF ${ }^{1}$, A. JÄCKEL ${ }^{1}$, E. KOPP ${ }^{1}$, U. LANGER ${ }^{1}$, M. MILDNER ${ }^{1}$, J. MÜLLER ${ }^{1}$,

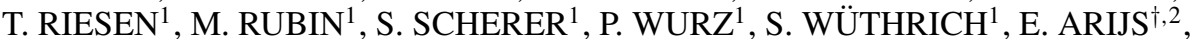 \\ S. DELANOYE ${ }^{2}$, J. DE KEYSER ${ }^{2}$, E. NEEFS ${ }^{2}$, D. NEVEJANS ${ }^{2}$, H. RÈME $^{3}$, \\ C. AOUSTIN ${ }^{3}$, C. MAZELLE ${ }^{3}$, J.-L. MÉDALE ${ }^{3}$, J.A. SAUVAUD ${ }^{3}$, \\ J.-J. BERTHELIER ${ }^{4}$, J.-L. BERTAUX ${ }^{4}$, L. DUVET ${ }^{4}$, J.-M. ILLIANO ${ }^{4}$, \\ S.A. FUSELIER ${ }^{5}$, A.G. GHIELMETTI ${ }^{5}$, T. MAGONCELLI ${ }^{5}$, E.G. SHELLEY ${ }^{5}$,

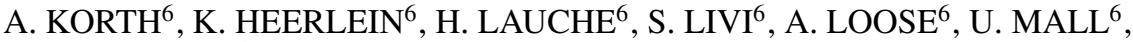 \\ B. WILKEN ${ }^{\dagger}$, F. GLIEM ${ }^{7}$, B. FIETHE ${ }^{7}$, T.I. GOMBOSI ${ }^{8}$, B. BLOCK ${ }^{8}$, \\ G.R. CARIGNAN ${ }^{8}$, L.A. FISK ${ }^{8}$, J.H. WAITE ${ }^{9}$, D.T. YOUNG ${ }^{9}$ and H. WOLLNIK ${ }^{10}$ \\ ${ }^{1}$ Physikalisches Institut, Universität Bern, CH-3012 Bern, Switzerland \\ ${ }^{2}$ Belgian Institute for Space Aeronomy (BIRA), Ringlaan 3, B-1180 Brussels, Belgium \\ ${ }^{3}$ CESR, F-31028 Toulouse cedex 4, France \\ ${ }^{4}$ IPSL, F-94100 Saint Maur, France \\ ${ }^{5}$ Lockheed Martin Advanced Technology Center, 3251 Hanover Street, Palo Alto, CA 94394, U.S.A. \\ ${ }^{6}$ MPI für Sonnensystemforschung, D-37191 Katlenburg-Lindau, Germany \\ ${ }^{7}$ Technische Universität, D-38106 Braunschweig, Germany \\ ${ }^{8}$ University of Michigan, Space Physics Research Laboratory, Ann Arbor, MI 48109, U.S.A. \\ ${ }^{9}$ Southwest Research Institute, POD 285 10, San Antonio, TX 78228, U.S.A. \\ ${ }^{10}$ University of Giessen, Physik Institut, D-35392 Giessen, Germany \\ (*Author for correspondence: E-mail: altwegg@space.unibe.ch)
}

(Received 8 February 2006; Accepted in final form 31 May 2006)

\begin{abstract}
The Rosetta Orbiter Spectrometer for Ion and Neutral Analysis (ROSINA) will answer important questions posed by the mission's main objectives. After Giotto, this will be the first time the volatile part of a comet will be analyzed in situ. This is a very important investigation, as comets, in contrast to meteorites, have maintained most of the volatiles of the solar nebula. To accomplish the very demanding objectives through all the different phases of the comet's activity, ROSINA has unprecedented capabilities including very wide mass range ( 1 to $>300 \mathrm{amu}$ ), very high mass resolution ( $m / \Delta m>3000$, i.e. the ability to resolve $\mathrm{CO}$ from $\mathrm{N}_{2}$ and ${ }^{13} \mathrm{C}$ from ${ }^{12} \mathrm{CH}$ ), very wide dynamic range and high sensitivity, as well as the ability to determine cometary gas velocities, and temperature. ROSINA consists of two mass spectrometers for neutrals and primary ions with complementary capabilities and a pressure sensor. To ensure that absolute gas densities can be determined, each mass spectrometer carries a reservoir of a calibrated gas mixture allowing in-flight calibration. Furthermore, identical flight-spares of all three sensors will serve for detailed analysis of all relevant parameters, in particular the sensitivities for complex organic molecules and their fragmentation patterns in our electron bombardment ion sources.
\end{abstract}

Keywords: comet, coma composition, mass spectrometry, Rosetta

Abbreviations: ADC, analogue-to-digital converter; ASP, acceleration supply pack; CASYMIR, calibration system for the mass spectrometer instrument ROSINA; CEM, channel electron multiplier; COPS, comet pressure sensor; DFMS, double focusing magnetic

Space Science Reviews (2007) 128: 745-801

DOI: $10.1007 / \mathrm{s} 11214-006-8335-3$

(C) Springer 2007 
mass spectrometer; DPU, data processing unit; EGSE, electrical ground support equipment; ESA, electroStatic analyzer; ETS, equivalent time sampling; ETSL, equivalent time sampling light; FC, Faraday cup; FDP, floating detector pack; FEC, filament emission controller; FIFO, first in first out; FM, flight model; FOV, field of view; FS, flight spare model; GCU, gas calibration unit; HV, high voltage; IMS, ion mass spectrometer on Giotto; LEDA, linear electron detector array; LVPS, low voltage power supply; MCP, multichannel plate; MCP, main controller; MEP, main electronics pack; MLI, multiLayer insulation; MS, mass spectrometer; NMS, neutral mass spectrometer on Giotto; RDP, remote detector pack; ROSINA , Rosetta Orbiter Spectrometer for Ion and Neutral Analysis; RTOF, reflectron type time-of-flight mass spectrometer; SEL, single event latch-up; SEU, single event upset; TDC, time-to-digital converter; TIMAS, toroidal imaging mass angle spectrograph; TOF , time of flight; UHV, ultra-high vacuum

\section{Introduction}

Comets are believed to be the most pristine bodies in the solar system. They were created 4600 million years ago far away from the sun and have remained for most of the time of their existence far outside of Pluto's orbit. They are small enough to have experienced almost no internal heating. They therefore present a reservoir of well-preserved material from the time of the Solar System's creation. They offer clues to the origin of the Solar System's material and to the processes that led from the solar nebula to the formation of planets. Some cometary material can even be traced back to the dark molecular cloud from which our Solar System emerged (e.g. Irvine, 1999). In contrast to meteorites (the other primitive material available for investigations), comets have retained the volatile part of the solar nebula.

Several interesting questions on the history of the Solar System materials can therefore be answered only by studying comets. In particular, the composition of the volatile material - the main goal of the ROSINA instrument. Below is a list of measurements still to be made and the associated science that can benefit from these measurements. The list is certainly incomplete and will evolve with time.

\section{Elemental abundances:}

- nitrogen abundance: physical and chemical conditions during comet formation;

- noble gases: processing of comets.

Isotopic abundances:

- $\mathrm{D} / \mathrm{H}$ in heavy organic molecules: origin of material;

- other isotopes in different molecules ( $\mathrm{C}, \mathrm{O}$ etc.): origin of material.

\section{Molecular abundances:}

- heavy organic molecules: origin of material; processing of material prior to incorporation in comets;

- reduced vs. oxidized molecules: chemical and physical conditions during molecule formation, origin of material; 
- series of molecules, e.g. $\mathrm{C}_{n} \mathrm{H}_{m}$ : origin of material, processing of material prior to incorporation in comets;

- $\mathrm{O}_{2}, \mathrm{O}_{3}$ : origin of terrestrial oxygen;

- radicals: physical and chemical conditions during comet formation, processing of comets.

Physical and chemical processes:

- extended sources: composition of dust in the coma;

- molecular abundances as function of heliospheric distance: nucleus composition, and processing of nucleus;

- molecular abundance differences in jets: homogeneity of nucleus composition; spatial and temporal differences;

- abundance differences between Oort cloud comets and Kuiper belt comets: physical and chemical conditions in the different comet forming regions, chemistry in the solar nebula and sub-nebulae.

\section{Scientific Goals}

As part of the core payload of the Rosetta mission, the Rosetta Orbiter Spectrometer for Ion and Neutral Analysis (ROSINA) is designed to answer outstanding questions posed by the Rosetta mission's main objectives. The spectrometer's primary objective is to determine the elemental, isotopic, and molecular composition of the comet's atmosphere and ionosphere, as well as the temperature and bulk velocity of the gas and the homogenous and inhomogeneous reactions of the gas and ions in the dusty cometary atmosphere and ionosphere.

In determining the composition of the atmosphere and ionosphere, the following prime scientific objectives, also set by the Rosetta Science Definition Team (ESA SCI (93)7), will be achieved:

- Determination of the global molecular, elemental, and isotopic composition and the physical, chemical, and morphological character of the cometary nucleus.

- Determination of the processes by which the dusty cometary atmosphere and ionosphere are formed, and characterization of their dynamics as a function of time, heliocentric and cometocentric position.

- Investigation of the origin of comets, the relationship between cometary and interstellar material and the implications for theories on the origin of the Solar System.

- Investigation of possible asteroid outgassing and establishing the relationships between comets and asteroids.

To accomplish these very demanding objectives, ROSINA must have unprecedented capabilities, including very wide mass range, from $1 \mathrm{amu}$ (hydrogen) to 
$>300 \mathrm{amu}$ (organic molecules); very high mass resolution $(m / \Delta m>3000)$ (ability to resolve $\mathrm{CO}$ from $\mathrm{N}_{2}$ and ${ }^{13} \mathrm{C}$ from ${ }^{12} \mathrm{CH}$ ); very wide dynamic range $\left(10^{10}\right)$ and high sensitivity $\left(>10^{-5} \mathrm{~A} / \mathrm{mbar}\right)$ to accommodate very large differences in ion and neutral gas concentrations and large changes in the ion and gas flux as the comet changes activity between aphelion and perihelion; and the ability to determine the outflowing cometary gas flow velocities. ROSINA monitors and characterizes the different phases of comet activity from aphelion through perihelion, thereby leading to a full understanding of cometary behavior. Correlated observations with, for example, the dust instruments, the magnetometer and the surface science package further augment the scientific return from ROSINA.

\section{Instrument requirements}

Table I lists the science objectives and the instrument requirements to achieve them. ROSINA's performance is summarized in Table II, and the comparison of operating ranges of the two mass analyzers is given in Figure 1. For comparison, the mass range of the Giotto Ion Mass Spectrometer IMS is given. The requirements listed in Table I are unprecedented in space mass spectrometry. So far, no single instrument can fulfill all of them. A three-sensor approach has therefore been adopted: each sensor is optimized for part of the scientific objectives while complementing the other sensors. In view of the very long mission, this approach also provides some redundancy.

DFMS is a double focusing magnetic mass spectrometer with a mass range 12$150 \mathrm{amu}$ and a mass resolution of 3000 at $1 \%$ peak height. It is optimized for very high mass resolution and large dynamic range;

$R T O F$ is a reflectron type time-of-flight mass spectrometer with a mass range 1 to $>300$ amu and a high sensitivity. The mass resolution is better than 500 at $1 \%$ peak height. It is optimized for high sensitivity over a very broad mass range;

COPS consists of two pressure gauges providing density and velocity measurements of the cometary gas.

This three-sensor approach had to fit within the mass and power budget allocated to ROSINA. An overview on the mechanical dimensions, mass, and power for the three sensors is given in Table III.

\section{The ROSINA Instrument Package}

\section{DFMS}

Design Goals

The double focusing mass spectrometer is a state-of-the-art high-resolution Mattauch - Herzog (Mattauch and Herzog, 1934) mass spectrometer (resolution 
TABLE I

Science objectives and measurement requirements for ROSINA

\begin{tabular}{|c|c|c|}
\hline Scientific objectives & $\begin{array}{l}\text { Associated critical } \\
\text { measurements }\end{array}$ & Measurement requirements \\
\hline $\begin{array}{l}\text { Determine elemental } \\
\text { abundances in the gas }\end{array}$ & Separate $\mathrm{CO}$ from $\mathrm{N}_{2}$ & $\begin{array}{l}\text { Mass resolution }>2500 \text { at } 1 \% \\
\text { of peak height at mass } \\
28 \mathrm{amu}\end{array}$ \\
\hline $\begin{array}{l}\text { Determine molecular } \\
\text { composition of volatiles }\end{array}$ & $\begin{array}{l}\text { Measure and separate heavy } \\
\text { hydrocarbons (neutrals and } \\
\text { ions) up to mass } 300 \mathrm{amu}\end{array}$ & $\begin{array}{l}\text { Mass range } 1 \text { to }>300 \mathrm{amu} \\
\text { with a resolution of }>300 \\
\text { at } 1 \% \text {; sensitivity } \\
>10^{-3} \mathrm{~A} / \mathrm{mbar}\end{array}$ \\
\hline $\begin{array}{l}\text { Determine isotopic } \\
\text { composition of volatiles }\end{array}$ & $\begin{array}{l}\text { Separate }{ }^{12} \mathrm{CH} \text { and }{ }^{13} \mathrm{C} \text {. } \\
\text { Measure HDO, DCN, and } \\
\text { other deuterated neutrals } \\
\text { and ions }\end{array}$ & $\begin{array}{l}\text { Mass resolution }>3000 \text { at } 1 \% \\
\text { peak height, relative } \\
\text { accuracy } 1 \%, \text { absolute } \\
\text { accuracy } 10 \%\end{array}$ \\
\hline $\begin{array}{l}\text { Study the development of the } \\
\text { cometary activity }\end{array}$ & $\begin{array}{l}\text { Measure the composition } \\
\text { (water and minor } \\
\text { constituents) between } \\
\text { 3.5 AU (gas production rate } \\
10^{24} \mathrm{~s}^{-1} \text { ) and perihelion } \\
\left(10^{29} \mathrm{~s}^{-1}\right)\end{array}$ & $\begin{array}{l}\text { Mass range } 1 \text { to }>300 \mathrm{amu}, \\
\text { dynamic range } 10^{8}\end{array}$ \\
\hline $\begin{array}{l}\text { Study the coma chemistry } \\
\text { and test existing models }\end{array}$ & $\begin{array}{l}\text { Measure ions and molecules } \\
\text { in the mass range } \\
1-300 \mathrm{amu} \text { and their } \\
\text { velocity and temperature }\end{array}$ & $\begin{array}{l}\text { Mass range for ions and } \\
\text { neutrals } 1 \text { to }>300 \mathrm{amu}, \\
\text { dynamic range } 10^{8}, \\
\text { sensitivity }>10^{-3} \mathrm{~A} / \mathrm{mbar}\end{array}$ \\
\hline $\begin{array}{l}\text { Study the gas dynamics and } \\
\text { the interaction with the dust }\end{array}$ & $\begin{array}{l}\text { Measurement of the bulk } \\
\text { velocity and temperature of } \\
\text { the gas }\end{array}$ & $\begin{array}{l}\text { Bulk velocity corresponding } \\
\text { to } E=0.02 \mathrm{eV} \pm 10 \% \\
\text { temperature }=0.01 \mathrm{eV} \\
\pm 20 \%\end{array}$ \\
\hline $\begin{array}{l}\text { Characterization of the } \\
\text { nucleus }\end{array}$ & $\begin{array}{l}\text { Characterization of outbursts } \\
\text { and jets of limited angular } \\
\text { extent }\end{array}$ & $\begin{array}{l}2^{\circ} \text { Narrow field of view, time } \\
\text { resolution }=1 \mathrm{~min}\end{array}$ \\
\hline Characterization of asteroids & $\begin{array}{l}\text { Detect asteroid exosphere or } \\
\text { determine upper limit }\end{array}$ & $\begin{array}{l}\text { Extreme sensitivity for } \mathrm{H}_{2} \mathrm{O} \text {, } \\
\mathrm{CO} \text {, and } \mathrm{CO}_{2}\end{array}$ \\
\hline
\end{tabular}

$m / \Delta m>3000$ at $1 \%$ peak height) with a high dynamic range of $10^{10}$ and a good sensitivity of $10^{-5} \mathrm{~A} / \mathrm{mbar}$. It is based on well-proven design concepts, which were optimized for mass resolution and dynamic range using modern methods for calculating ion optical properties. The main design goals are given in Table II.

The DFMS has two basic operation modes: a gas mode for analyzing cometary gases and an ion mode for measuring cometary ions. Switching between the gas and ion modes requires a change of only a few potentials in the ion source and suppression of the electron emission that is used to ionize the gas. All other operations are identical in the two modes. 


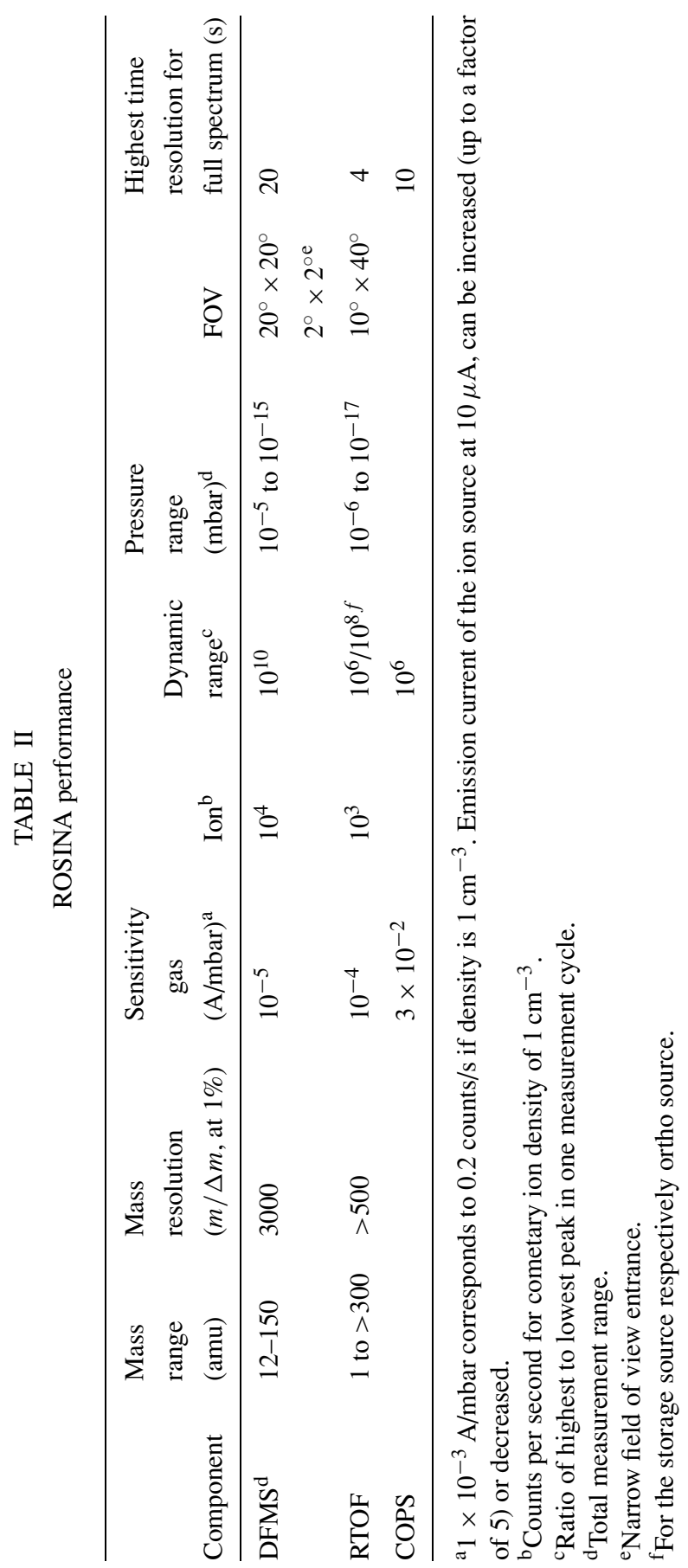


TABLE III

ROSINA mass and power budget

\begin{tabular}{lcll}
\hline Unit & Mechanical dimensions $(\mathrm{cm})$ & Mass $(\mathrm{kg})$ & Mean power $(\mathrm{W})$ \\
\hline DFMS & $63 \times 63 \times 26$ & 16.2 & 19 \\
RTOF & $114 \times 38 \times 24$ & 14.7 & 24 \\
COPS & $26 \times 26 \times 17$ & 1.6 & 3 \\
DPU & $16 \times 14 \times 14$ & 2.3 & 3 \\
Total & & 34.8 & 49 \\
\hline
\end{tabular}
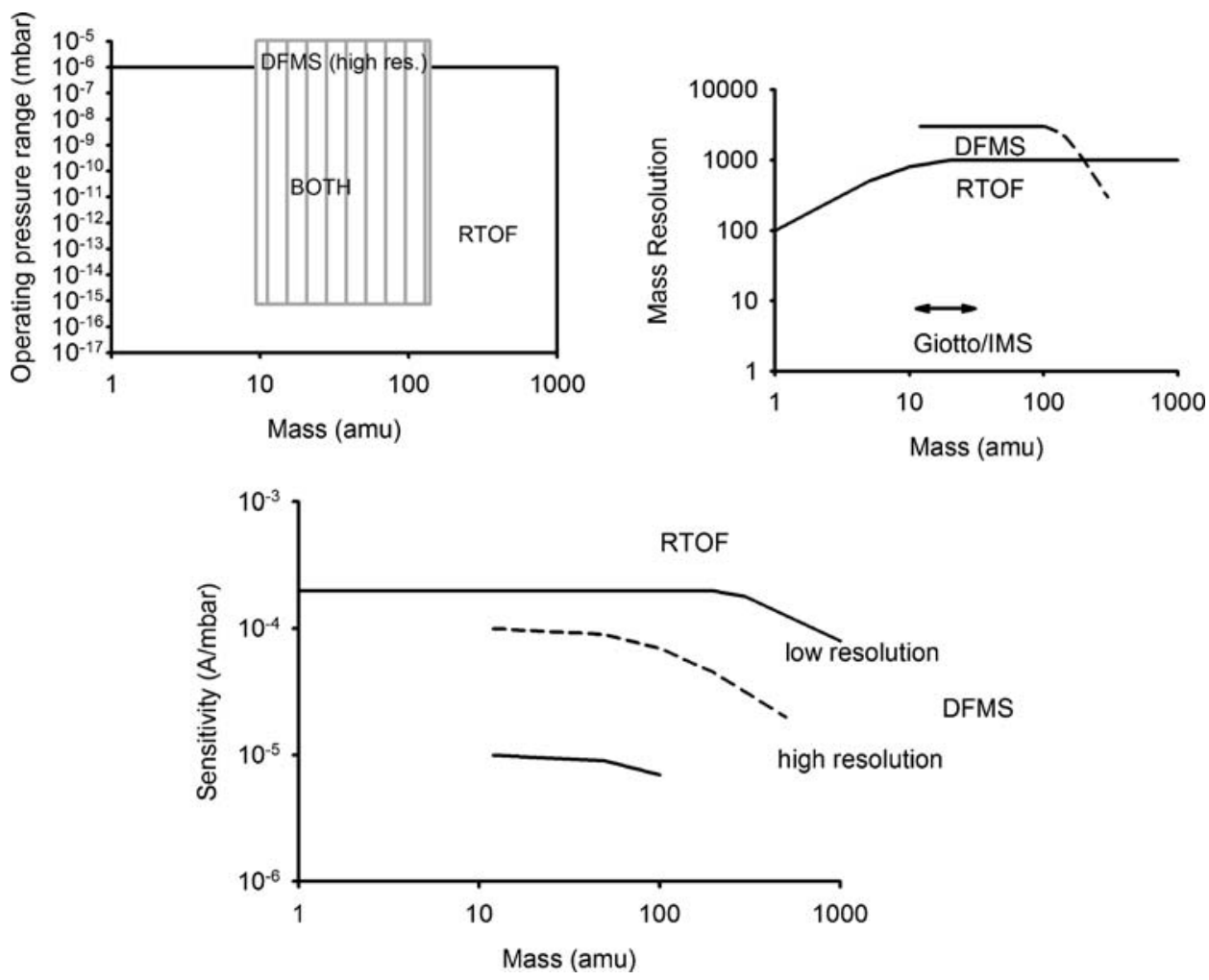

Figure 1. Comparison of the operating ranges of DFMS and RTOF. The mass range of the Ion Mass Spectrometer IMS flown on the Giotto mission is also given.

\section{Ion Optics}

Ion source: The design of the ion source is based on the electron bombardment source used in modern laboratory rare gas mass spectrometers. This source combines high sensitivity $\left(10^{-3} \mathrm{~A} / \mathrm{mbar}\right)$ with good linearity over a very wide gas pressure range (from several $10^{-5}$ mbar to below $10^{-14} \mathrm{mbar}$ ), small energy dispersion, and low background. 


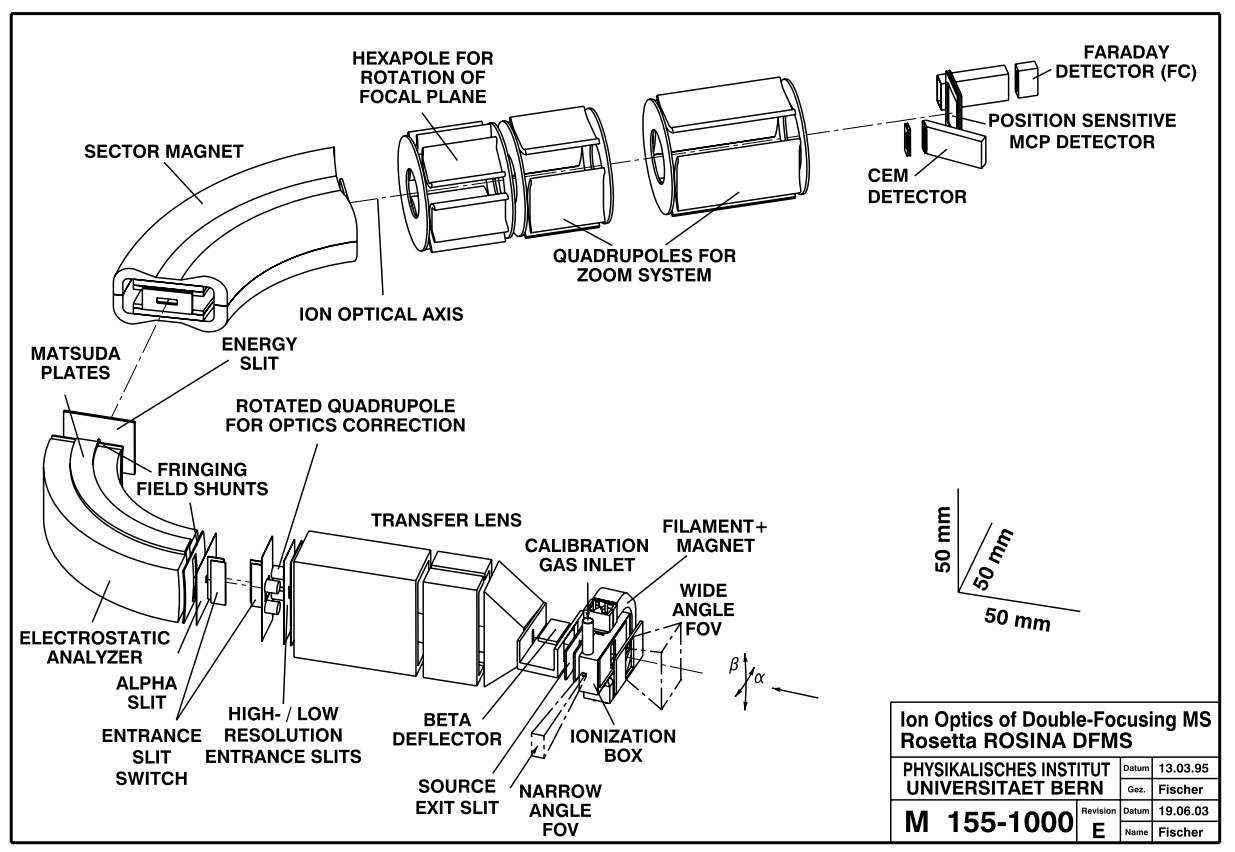

Figure 2. Three-dimensional view of the main ion optical elements of DFMS.

The source has two viewing directions with different field of views (FOV). The one parallel to the source axis has a wide FOV of $\pm 20^{\circ}$, the one orthogonal to it has a narrow FOV of $\pm 2^{\circ}$. Most of the measurements will use the wide FOV, allowing cometary gas with wide angular spread in the flow direction to enter the ionization region. The narrow FOV will be used for determining the exact flow direction of the cometary gas-jets. The axis of the wide FOV is parallel to the axis of the cameras, and normally directed towards the nucleus.

The FOVs are determined by a set of electrodes upstream of the ionization region shown in Figure 2. Suitable potentials applied to these electrodes prevent the entry of low-energy ambient ions into the DFMS operating in the gas mode. Cometary ions with higher energies $(>60 \mathrm{eV})$ cannot pass through the analyzer and it is not necessary to prevent their entry into the ion source. In the ion mode, the potentials on these electrodes are changed to attract the cometary ions even in case of positive charging of the $\mathrm{S} / \mathrm{C}$ and to focus them into the gas ionization region of the source. A negatively biased coarse meshed grid surrounding the ion source area up to a distance of $15 \mathrm{~cm}$ is used to augment the ion sensitivity.

The instrument outgassing could lead to serious interference while measuring the cometary gases. To keep the interference as low as possible, the entire ion source region is built to ultra-high vacuum (UHV) standards and degassed before launch and also during flight. Since the narrow analyzer entrance slit has a very 
low vacuum conductance (the only connection between the source and analyzer regions), outgassing from internal sensor parts is efficiently suppressed.

The cometary gases entering the source are ionized with an electron beam parallel to the slit direction. A weak magnetic field $(0.02 \mathrm{~T})$ is used to guide the electrons. Two filaments are provided to give redundancy. The electron energy can be varied between about 10 and $90 \mathrm{eV}$. At higher electron energies $(>60 \mathrm{eV})$, the ionization cross section is maximal and hence the instrument sensitivity is at its optimum. At low electron energies, the cross section is reduced but there is much less fragmentation of the more complex molecules. This can be used to facilitate the identification of unknown species. The ion source can be operated with electron currents of 2 , 20 , or $200 \mu \mathrm{A}$ to provide three sensitivity levels which differ by a factor of 10 . By means of a small gas tube, calibrated amounts of a gas mixture can be leaked into the ionization region and will be used for in-flight tests and calibration of the DFMS.

A mass scan is achieved by varying the ion energy. To minimize mass and sensitivity discrimination, the ion source is operated at a fixed acceleration potential of $1 \mathrm{kV}$. After the first focus point (line width typical $150 \mu \mathrm{m}$ ), a transfer lens is used to accelerate and/or decelerate and focus the ions onto the entrance slits of the analyzer section. Two entrance slits are used, a narrow slit $(14 \mu \mathrm{m})$ and a wide slit $(200 \mu \mathrm{m})$. The ion beam can be guided through the narrow slit in the high-resolution mode or through the wide slit in the low-resolution mode by electrostatic deflection. The axis of the transfer lens is tilted by $6^{\circ}$ relative to the ion source axis to protect the narrow entrance slit of the analyzer from cometary dust particles.

The final ion energy is established in the transfer section of the ion source. To pass through the analyzer with its fixed magnetic field, the ion energy must be changed from $6 \mathrm{keV}$ at mass $12 \mathrm{amu}$ to $430 \mathrm{eV}$ at $140 \mathrm{amu}$. Thus, the $1 \mathrm{keV}$ ions from the source are either accelerated or decelerated in this section and at the same time focused on the entrance slit of the analyzer.

The mass analyzer: For the Rosetta DFMS, the following key requirements were considered to optimize the analyzer geometry:

- Mass resolution $m / \Delta m>3000$ for a mass range $12-150 \mathrm{amu} / \mathrm{q}$ at the $1 \%$ peak level.

- Good energy-focusing properties to allow $\Delta E / E$ up to $1 \%$ (important if lower ion energies are used).

- High mass dispersion to allow the use of a position-sensitive focal plane detector.

- A large free viewing angle (preferably $2 \pi$ ) for the ion source acceptance.

- A small overall analyzer size and a radius of curvature in the magnet below $10 \mathrm{~cm}$.

The resulting optimal field geometry is a combination of a $90^{\circ}$ toroidal electrostatic analyzer (ESA) with a $60^{\circ}$ sector magnet for momentum analyses (see 
Figure 2). High mass dispersion can be achieved by using an electrostatic zoom lens system.

At high mass resolutions, detector and focal plane coincide only at one specific mass number either in the center of the multichannel plate (MCP) or at the channel electron multiplier (CEM) detector. High resolution can thus only be obtained for the mass multiplets at one mass number and the mass lines from neighboring mass numbers will show less mass resolution. To obtain a full high resolution mass spectrum from 12 to $150 \mathrm{amu} / \mathrm{q}$, it is thus necessary to record a mass spectrum at each integer mass number.

The analyzer can also be operated in a low-resolution mode, which allows the simultaneous recording of several mass lines on the position-sensitive detector with a resolution of $m / \Delta m$ of several hundred. Neighboring integer mass numbers are well separated at this mass resolution. In this mode, the zoom system is used to rotate the focal plane into the plane of the position-sensitive detector.

Ion detectors : The instrument has three independent ion detectors (see Figure 2).

Design considerations for detectors: A relatively small and well-defined mass range from a specific setting of the ion optical parameters is focused in the focal plane and a one-dimensional image is obtained. The detector package, which has been designed specifically for the DFMS, uses three different detectors at the focal plane. The detectors have to meet a number of requirements, which are summarized as follows:

- In the central part of the ion beam, the detector must provide an image at the focal plane with a resolution corresponding to the highest mass resolving capability of the spectrometer. This corresponds to a maximum pixel width of $25 \mu \mathrm{m}$ in the direction of the focal plane over a length of about $1.25 \mathrm{~cm}$.

- The overall dynamical range of the detector must be able to cover the extremely large variations of ion fluxes. These changes arise predominantly from the variations of nucleus outgassing as a function of comet activity, from the large differences in density between major constituents, such as water and minor constituents or isotopes, and also from the varying sensitivity of the instrument as a function of its mode of operation (ion and neutral mode, low or high mass resolution, etc.). The realistic and technically possible overall dynamical range was estimated to be 10 orders of magnitude.

- The instantaneous dynamical range has to cope with the temporal variations of the cometary gas during a single measurement and with the differences in ion fluxes impinging at various locations on the detector front face within the range of masses measured simultaneously. In view of the expected quite slow temporal variations of the cometary atmosphere in the vicinity of the orbiter and to the fast measuring modes (as fast as 100 measurements per second), the second constraint is certainly more important. From the anticipated chemical 
and isotopic composition of the cometary gas, an instantaneous dynamical range of $4 \times 10^{3}$ was used as design objective.

- The detector package has to guarantee reliable measurements up to the end of a long mission of more than 10 years. Possible degrading of the gain of MCPs or channeltrons requires the possibility of an absolute calibration by measuring directly the ion fluxes in the focal plane that correspond to the most abundant species as water and its ion group. To achieve this absolute calibration, a Faraday cup (FC) is included beside the main imaging, MCP based, detector and instrument modes move the water peak alternately from the imaging detector to the FC.

Reliability considerations for this long and certainly innovative mission have finally led to two final requirements. First, it was decided to equip the detector package with a third detector, allowing measurements of the mass spectrum with a resolution and a dynamical range which would be similar to those provided by a single $25 \mu \mathrm{m}$ pixel of the imaging detector. This was achieved by using a channeltron (CEM) with a $20 \mu \mathrm{m}$ slit in front of the entrance to insure the necessary resolution. The second requirement is related to the imaging detector itself; the large height of the mass focal lines in the focal plane permits splitting of the collector of the imaging detector into two halves, one above and another below the plane of symmetry of ion trajectories, offering two separate and redundant collectors and electronics, and thus ensuring a total redundancy of this critical part of the instrument.

Description of the detector package: The detector package is shown in Figure 3 and represents a cut in the plane of symmetry of the ion trajectories. The dark line indicates the location of the theoretical focal plane of the spectrometer. The main imaging detector is located in the center of the detector package, as indicated by the position of the two Chevron MCPs. The Chevron MCPs with a rectangular form were adapted to the geometry of the focal plane. Its pore size is $6 \mu \mathrm{m}$, the inclination of its tubes $13^{\circ}$, and the maximum total gain at saturation is about $10^{6}$. In order to keep the maximum resolution, the MCP front face should be located exactly at the focal plane. However, the energy of the ions collected on the front face of the MCP should be larger than $\sim 1 \mathrm{keV}$ in order to guarantee a large enough MCP detection efficiency. For this reason, the front face of the MCP can be biased up to a negative post-acceleration voltage of $-3 \mathrm{kV}$. In order to prevent large perturbations of the ion trajectories, which would totally deteriorate the focusing properties of the spectrometer, the MCP must be approximately perpendicular to the average ion trajectories and positioned as shown in Figure 3. Extensive numerical modeling has shown that with such a geometry, global resolution of the instrument is adequate and reaches the specified value.

The CEM is located at the upper left part of the detector package. A $20 \mu \mathrm{m}$ wide slit is positioned about $1 \mathrm{~cm}$ ahead of it and coincides with the location of the end of 


\section{IONS}

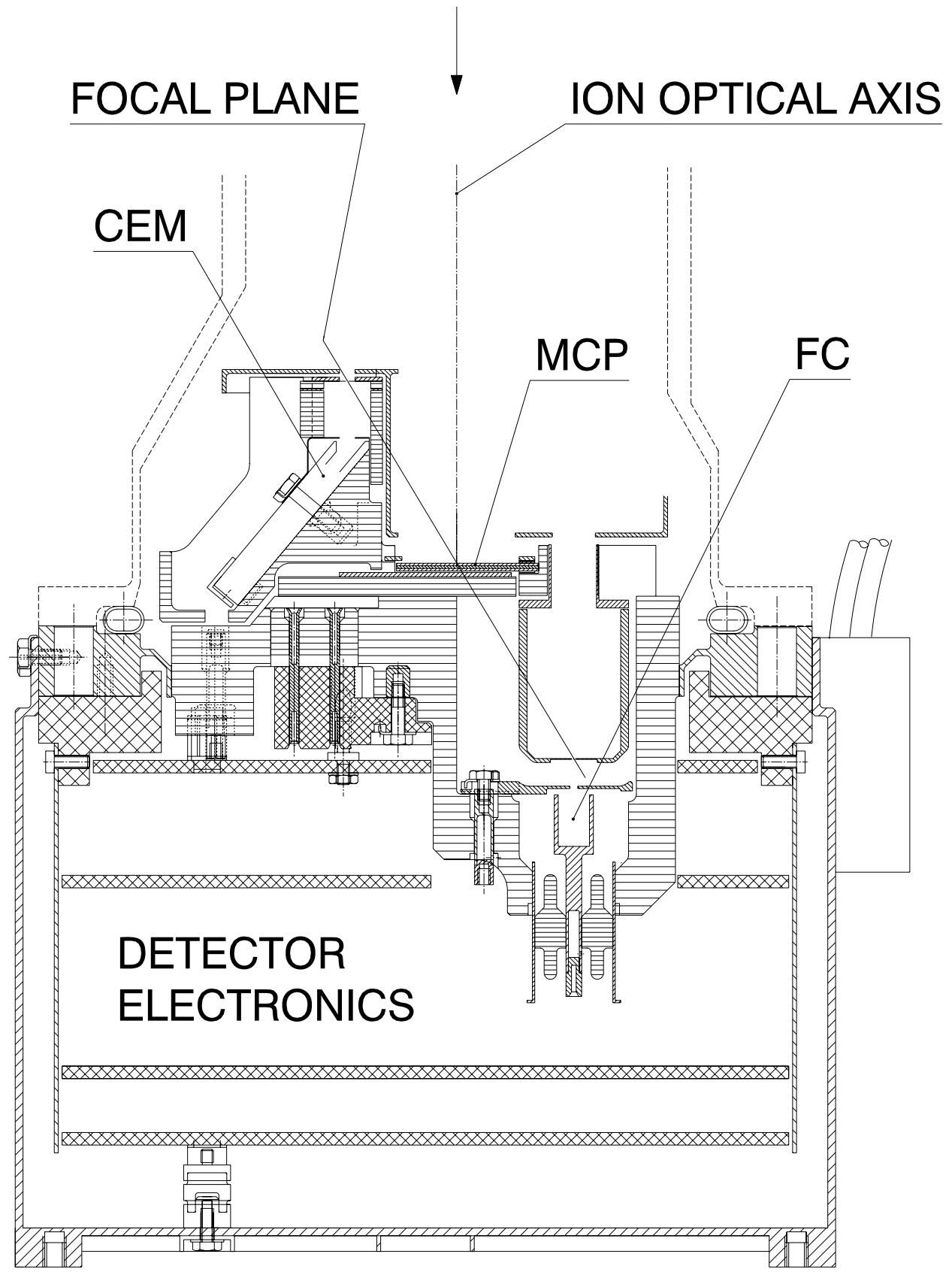

Figure 3. Section of the detector package in the plane of symmetry with the associated RDP electronics boards. FC: Faraday cup, MCP: multi-channel plate with LEDA512, CEM: channeltron. The focal plane has an angle of $31^{\circ}$ relative to the ion optical axis. 


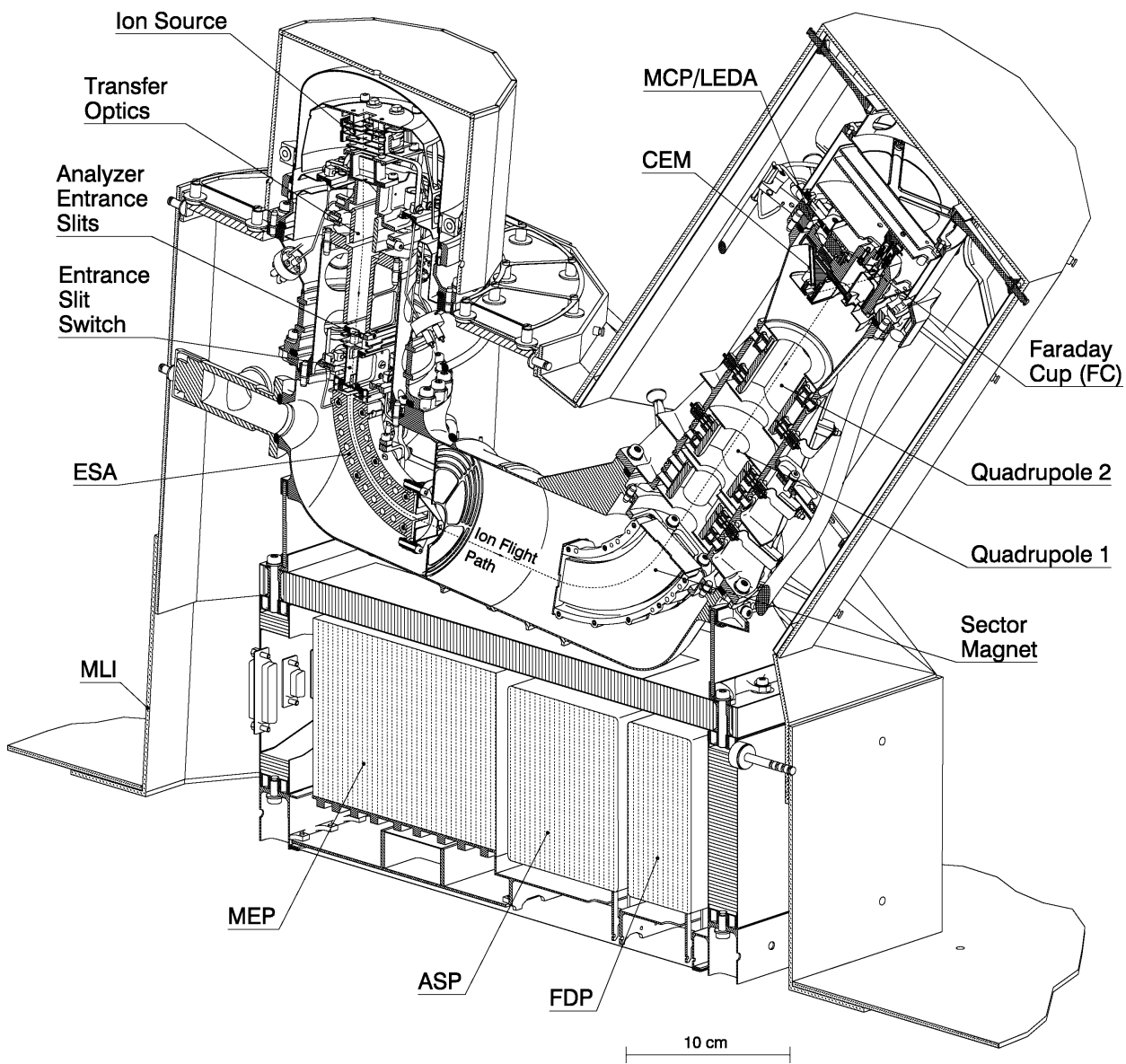

Figure 4. A three-dimensional view of the DFMS sensor with the cover opening mechanism and the thermal hardware.

the focal plane. At the same time, it prevents the high voltage on the CEM entrance to leak out and effect ion trajectories in the drift space before the focal plane. The CEM may be operated in a counting and an analog mode.

The FC can be seen in the right end of Figure 3 with a $0.35 \mathrm{~mm}$ wide slit in front of the cup and coincident with the right end of the focal plane. It provides the capability of absolute instrument and detector calibration and the medium resolution measurements of the water peak in a current range $10^{-14}$ to $10^{-8} \mathrm{~A}$.

\section{Mechanical/Structure}

Figure 4 shows a three-dimensional picture of the DFMS sensor. The main components are the primary structure containing the ion optics, the secondary structure containing the electronics, the cover opening mechanism, and the in-flight gas calibration unit (GCU). 
Titanium and ceramics are used in the primary structure in order to reach the UHV requirements. The primary structure can be baked out up to $120^{\circ} \mathrm{C}$, or up to $250^{\circ} \mathrm{C}$ for the ion source alone. The banana-shaped tube contains all the ion optical elements. The mechanical requirements with respect to tolerances are extreme. The toroidal surfaces of the electrostatic analyzer have to be machined within $\pm 2 \mu \mathrm{m}$ of the ideal surface. All the ion optical elements are co-aligned with an accuracy of a few microns. Along the ion trajectories, all the surfaces are gold plated or gold sputtered in order to reach uniform surface potentials. The primary structure is electrically and thermally isolated from the secondary structure. The main part is operational up to $6 \mathrm{kV}$, whereas the entrance part with the ion source remains at a few volts relative to the spacecraft potential. The electrical insulation between the two parts is guaranteed by a ceramic ring. In order to maintain the detectors within the given temperature limits of -20 to $+30^{\circ} \mathrm{C}$, the black multilayer insulation (MLI) surrounding the detector part contains a non-operational heater as well as a dedicated radiator for cooling.

Prior to launch, the primary structure was baked out and then sealed by a cover to minimize contamination. It was evacuated through a pump-off valve. A vacuum requirement of $<10^{-5}$ mbar after 1 week without pumping was imposed on this sealed structure. The cover was first opened in space by a pyrotechnical device. After initial opening, the cover can be closed to protect the instrument against undesired ambient gas flow like, e.g., thruster exhaust. It is intended to close the cover during thruster firing and in case of high dust activity near the comet to keep the sensor clean. In case of a failure of the cover motor, the gear of the cover can be disengaged with a second pyrotechnical device and the cover will then remain in an open position.

The secondary structure is made from aluminum, partly as honeycomb structure. It houses the different electronics subunits. The part of the electronics which is on high voltage is insulated from spacecraft ground by $\mathrm{BeO}$-standoffs in order to guarantee a good electrical insulation, at the same time good thermal conductivity. The primary structure is mounted on spring blades made from carbon fiber material on top of the secondary structure. This allows compensation for the different thermal expansion coefficients.

The in-flight calibration unit (GCU) contains two gas reservoirs of about 10 $\mathrm{cm}^{3}$ each of a noble gas and $\mathrm{CO}_{2}$ mixture at 5-6 bar pressure. It is identical to the one used in RTOF. Figure 5 shows the flight model of DFMS without thermal hardware.

\section{Electronics}

The ROSINA DFMS electronics described here provides power and controls the cover mechanism, the ion source and GCU, all elements of the ion optics, and the detectors. The instrument control is provided through an interface with the ROSINA DPU. Commanding and acquiring of housekeeping and science data is done by the ROSINA DPU. The DFMS electronics does not need to store data or commands. 


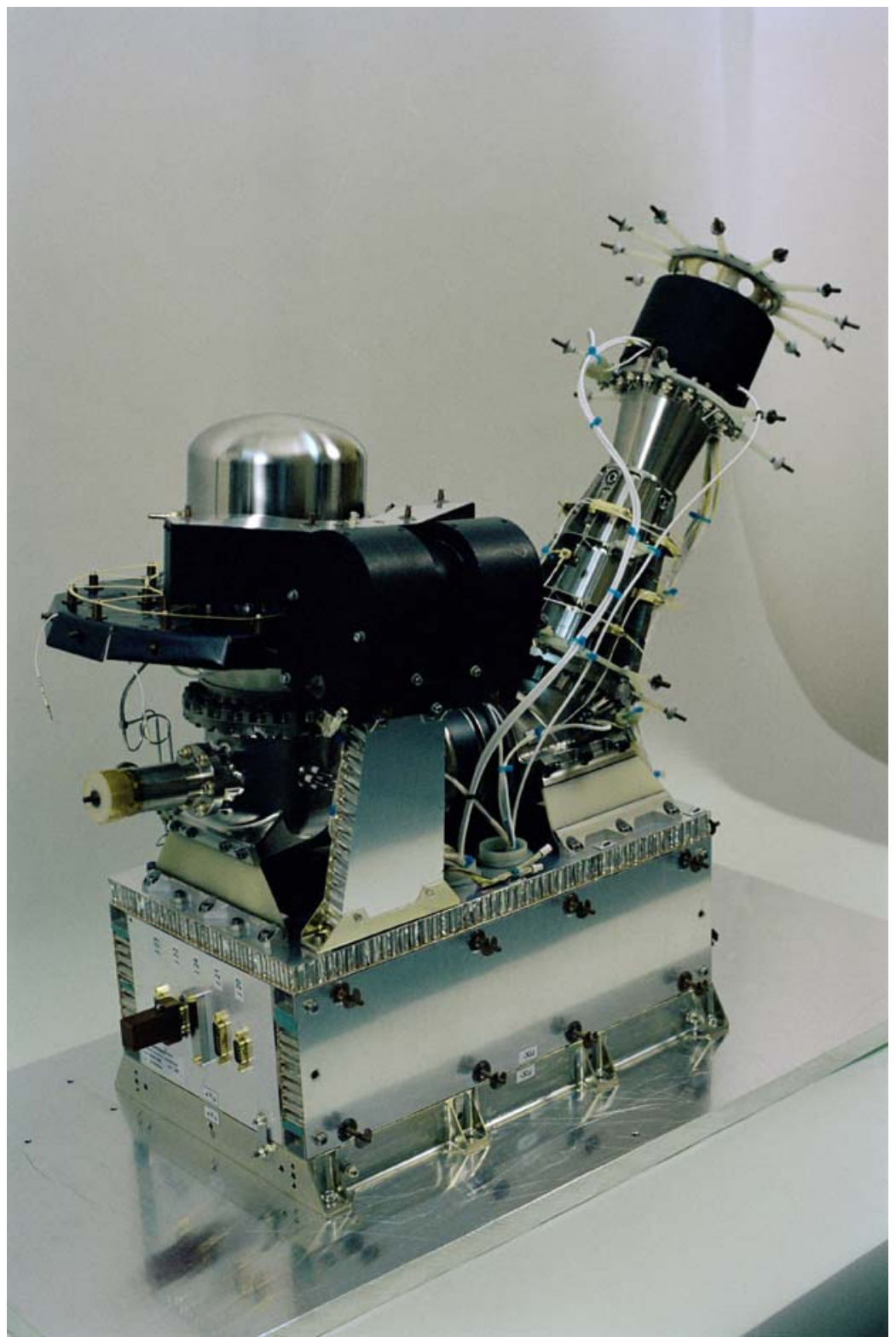

Figure 5. Flight model of DFMS without thermal hardware.

An overall block diagram is given in Figure 6 for the ion source and transfer optics and in Figure 7 for the analyzer and detectors.

The ion source is protected by its cover. Once the vacuum seal is broken after launch, it can be opened and closed and placed in intermediate positions. This capability is required to protect the instrument from contamination (for example, from 


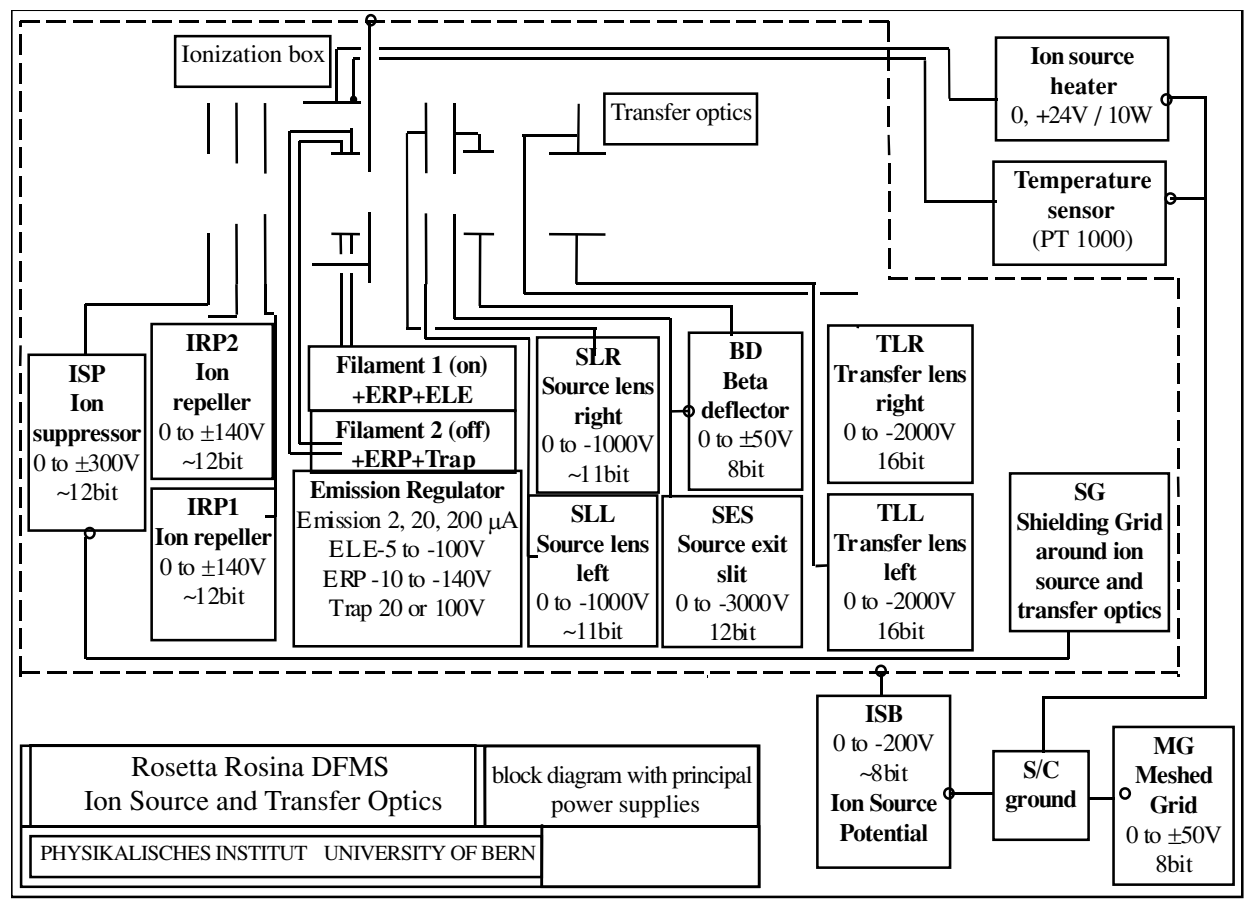

Figure 6. DFMS ion source and transfer optics block diagram.

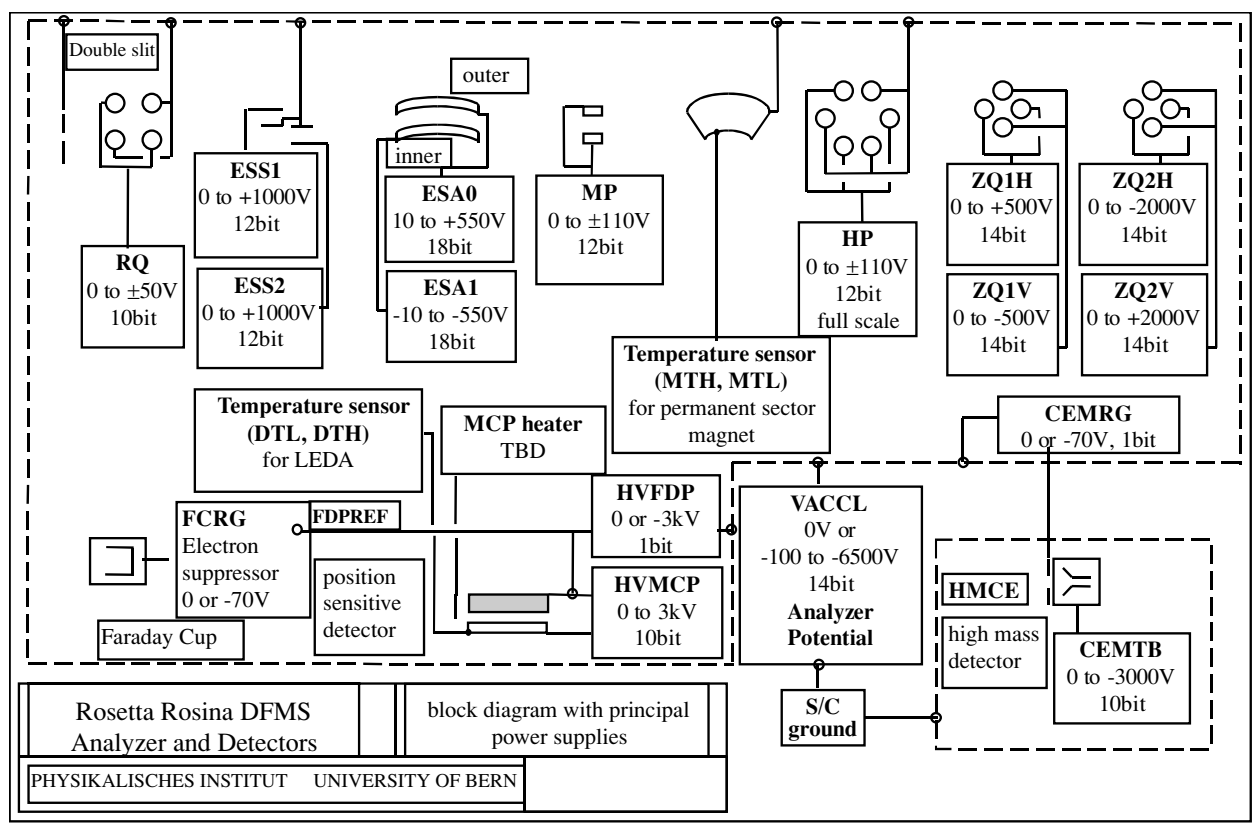

Figure 7. DFMS electronics block diagram for the analyzer section. 
very high pressures near the comet) and it provides a shutter, which can be partially closed, blocking the cometary ion and neutral influx. This second feature will allow in-flight calibration and a determination of the residual gas in the spectrometer.

The cover motor and the ion source are on spacecraft ground potential. The motor is controlled by a pre-programmed Actel chip which provides the capability to ramp up and down the cover motor current at selected rates, and to maintain a constant current input to the motor. End switches on the cover provide the motor controller with the open and close limits. In addition, the motor has Hall sensors used by the motor controller to count the number of motor revolutions. The position of the cover as a function of the number of revolutions was calibrated prior to launch and the cover can be placed in any arbitrary position.

The ion source contains two filaments (for redundancy), which are powered by the ion source controller. The ion source controller regulates the current to the filaments and also receives housekeeping information on the filament current and temperature in the vicinity of the filament (see Figure 6 for a block diagram). Safety current limiters for the ion source were set prior to electronics integration. Otherwise, the filaments can be commanded to any current level within the limits of the ion source controller. In addition to the low-voltage electronics and the power supply for electron emission, 10 other power supplies are used for the ion source.

Starting at the entrance to the ion source, there is one ion source voltage commandable from 0 to $\pm 300 \mathrm{~V}$ with 12 bits accuracy. This voltage repels the ions coming from the comet. After the ion suppression grid, two power supplies provide voltages to prevent ions created in the ion source from escaping back through the entrance aperture. Another two power supplies provide the ionization box with potentials to accelerate the electrons from the filament. The ions formed in the ion source are extracted from the ionization region, accelerated, and sent through the transfer optics section using high voltages from additional five power supplies. Two of these power supplies in the transfer optics section require 0 to $-2000 \mathrm{~V}$ with 16 bit accuracy. The accuracy of all power supplies in the ion source and ion optics is determined by the mass resolution requirements of the DFMS.

After the transfer optics section, ions pass through a wide range of ion optical elements, which ultimately focus a mass dispersed ion beam onto several possible detectors including a high-resolution, position-sensitive detector. Since the ion source potentials are referenced to spacecraft ground, the ion optics in the analyzer must float at a high voltage acceleration potential $\left(V_{\mathrm{acc}}\right)$. This floating acceleration potential is provided by a 14 bit 0 to $-6500 \mathrm{~V}$ power supply. Because all the ion optical elements float at this high potential, they are also electrically isolated from the power supplies and instrument controllers that reside on ground. Communication to and from these isolated power supplies is provided by a serial interface across several fiber optics channels. The design for the fiber optics was adapted from a similar device used in the Toroidal Imaging Mass Angle Spectrograph (TIMAS) (Shelley et al., 1995). The power to these units is supplied across a high-voltage transformer. 
The electrically controlled double slit system is powered by two $1000 \mathrm{~V} 12$ bit power supplies located after the transfer optics. Following a corrective lens element accomplished by a pair of plates biased at low voltage $(0-50 \mathrm{~V})$, the ions enter the electrostatic analyzer (ESA). This analyzer is biased from two 10 to $550 \mathrm{~V} 18$ bit power supplies. This high accuracy is needed to select specific ion energies, to focus specific masses on the channel electron multiplier in the detector and to achieve a good peak shape in the CEM high-resolution scan.

The ESA voltages are temperature compensated using a preprogrammed lookup table in the ESA controller. The lookup table is preprogrammed during ESA voltage calibration to compensate the temperature changes in the voltage and keep the ESA voltage stable during the measurement cycle with stability approaching $4 \mathrm{ppm}$ (18 bit).

The ion beam is corrected first by a Matsuda plate pair (Matsuda and Fujita, 1975) controlled by 0 to $\pm 110 \mathrm{~V}$. After the passage through the magnet, three $0 \pm 50 \mathrm{~V}$ power supplies are used to bias three individual zoom lenses (one hexapole and two quadrupoles). The magnet is a static element in the ion path but the temperature is monitored by the DFMS electronics.

In case the optical elements in this section are not active, the DFMS remains in the low mass resolution mode and the mass dispersed ion beam impinges on the detector according to the optical steering. When the four optical elements (powered by two 0 to $\pm 500 \mathrm{~V} 14$ bit and two 0 to $\pm 2000 \mathrm{~V} 14$ bit supplies) are active, the DFMS is in the high mass resolution mode, and the ion beam that impinges on the chosen detector is considerably more dispersed in the transverse (mass) direction.

Through a high-voltage transformer interface, the DFMS electronics also provides high voltages to the CEM detector and its repeller grid, the repeller grid for the FC detector and to the front and back side of the MCPs. The MCP voltages are programmable to 10 bit accuracy to allow a safe detector switch-on and to program the required potential changes used in the MCP gain control.

The entire electronics package is housed below the DFMS optics (see Figure 4). Three packages are attached to the DFMS base plate. These packages consist of the main electronics pack (MEP), the acceleration supply pack (ASP), and the floating detector pack (FDP). A fourth package discussed with the sensor is called the remote detector pack (RDP).

The MEP consists of eight electronics boards; a motherboard connects these with a low voltage power supply board. These boards are all at the local spacecraft ground and are attached directly to the base plate for optimum thermal dissipation.

- MEP-A: Data and command handler, providing interface with the DPU.

- MEP-B: Ion source heater and calibration.

- MEP-C: Mechanism control, providing control of the cover and the CEM detector high voltage.

- MEP-D: CEM data electronics. 
- MEP-E: Ion acceleration bias supply, providing the $-6500 \mathrm{~V}$ floating potential for the ion optics.

- MEP-F: Filament supply, providing the ion source filament potential.

- MEP-G: Ion source controller, controlling the potentials in the ion source to accelerate and focus the ions.

- MEP-H: Transfer optics high voltage.

- MEP-K: Pre-regulator for the transfer optics high voltage power supplies.

- MEP-M: Motherboard which connects MEP-A through K (residing below the base plate).

- MEP-N: Low voltage power supply, which interfaces with the spacecraft power provided through the DPU.

The ASP consists of five electronics boards. This pack floats at the $V_{\text {acc }}$ potential and is electrically isolated from the base plate by high-voltage $\mathrm{BeO}$-standoffs. Thermal dissipation is accomplished through these standoffs as well as radiatively from the sides of the pack.

- ASP-A: Low to high voltage used to generate the voltage for the FDP package floating at a potential above the ASP package.

- ASP-B: Digital control for the ASP package, including the ESA power supply.

- ASP-C: ESA high voltage, providing the 18 bit ESA voltages.

- ASP-D: Medium to high voltage, providing voltages for the optical elements after the ESA including the zoom optics.

- ASP-E: Interface and power for the ASP pack, connected across a high-voltage interface to the low voltage power supply in the MEP pack.

Detector electronics : The very large dynamic range is achieved with an analog detector system. In this system, charges are accumulated and/or currents are measured on a collector at the exit of the MCP. In this analog mode, the gain of the MCP can be varied over more than 6 orders of magnitude using an appropriate adjustment of the supply voltage, as used, for example, on the NMS instrument of the ESA Giotto mission. This gain control is added to the normal dynamic range of the collector electronics itself and fulfills the specification indicated above. In order to meet the resolution requirement of $\sim 25 \mu \mathrm{m}$, a new ASIC chip, the LEDA512 (linear electron detector array) was developed (Nevejans et al., 2000, 2002; Berthelier et al., 2002). This chip integrates two identical but independent detector systems, each consisting of a collector under the form of a row of 512 anodes (or pixels) collecting the electrons emitted from the back face of the MCP and of the associated charge integration electronics. With a pixel width of $22 \mu \mathrm{m}$ and a $3 \mu \mathrm{m}$ separation between neighboring pixels, this collector has a width of $12.8 \mathrm{~mm}$ in the focal plane over a height of $8 \mathrm{~mm}$ and each mass peak covers approximately 6 pixels. The back face of the MCP is separated by a distance of $0.2 \mathrm{~mm}$ from the plane of the LEDA collector. In the standard mode of operation, the electrons exiting from the MCP 
are accelerated by a potential of $\sim 250 \mathrm{~V}$, limiting the spreading of the space charge and maintaining the required resolution. Each individual pixel operates as a floating electrode of a capacitor with its second electrode at ground; the capacitance of a pixel is approximately $4 \mathrm{pF}$. In the read-out sequence, which can be made as fast as $10 \mathrm{~ms}$ for the 512 pixels, each pixel is connected sequentially through an analog multiplexer to a charge amplifier. This amplifier provides at its output a pulse with an amplitude proportional to the amount of electron charge collected on each pixel. A 12 bit ADC then converts the pulse height into a 12 bit digital word stored in a spectrum accumulation register. This register is ultimately read out by the instrument DPU through an opto-coupler link.

As a consequence of the accelerating voltage applied to the front face of the MCP and of the variable HV polarization between the front and the back faces of the MCP which controls its gain, the LEDA is at a "floating detector package" potential (FDP), which can reach several kilovolt with respect to the DFMS reference level. In order to avoid leakage currents problems on the FC and difficulties associated with two different high voltages in the electronics installed in the detector package, the FC electronics is polarized at the same floating voltage as the LEDA. All together, the electronics of the detector package represented in Figure 8 consists of two main parts:

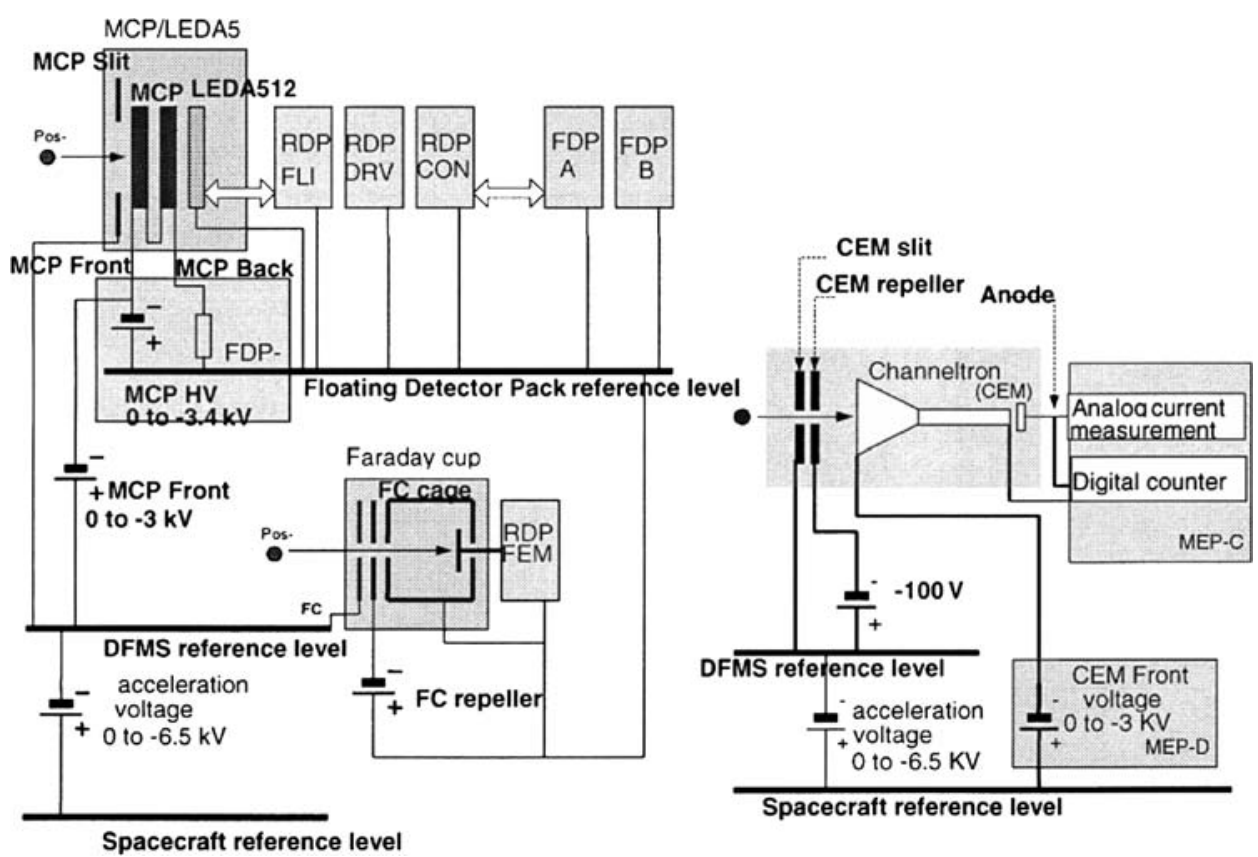

Figure 8. DFMS detector block diagram for MCP, CEM, and Faraday cup. 
- The remote detector package (RDP) has four boards located just behind the collector and the FC. They include the most sensitive circuits, which need to be placed as close as possible to the LEDA and the FC and the associated interface circuits with the FDP.

- The floating detector package (FDP) has three boards mounted on insulated standoffs on the DFMS base plate. They provide the digital interface with the RDP boards, process the analog signals from the LEDA and FC and deliver the MCP floating HV and FDP power supply voltages. This pack floats at the detector acceleration potential (somewhat above $V_{\text {acc }}$ of the ASP). Like the ASP, it is electrically isolated from the base plate by high-voltage standoffs and thermal dissipation is accomplished in the same way as the ASP dissipation.

- FDP-A: Analog processing for the high-resolution detector and the FC detector.

- FDP-B: Digital control for the detector.

- FDP-C: Interface and power for the FDP package, connected across a highvoltage interface to the low voltage power supply in the MEP pack.

\section{Measurement Sequences}

The instrument has a large number of operational parameters, which can be individually adjusted to fit any specific measurement requirements. However, a certain number of predetermined modes and measurement sequences are now implemented and it is expected that most measurements will use these modes. From time to time it will be necessary to retune voltages of the instrument to optimize the performance and to compensate for mechanical, thermal, etc. drifts which could occur in space. We expect that the basic retuning can be done autonomously, but some manual adjustments might still be necessary requiring extensive ground command sessions.

For any given instrument setting, we will use a basic integration time of approximately $20 \mathrm{~s}$ (MCP only). The accumulated spectra will be transferred to the DPU for further data processing. The adjustment of the instrument to a new setting, for instance a new value for the central mass, requires about $10 \mathrm{~s}$. This includes the time necessary to optimize the detector gain. A full high-resolution mass spectrum from 12 to $150 \mathrm{amu} / \mathrm{q}$ can thus be recorded in $79 \times 30 \mathrm{~s}=2370 \mathrm{~s}=40 \mathrm{~min}$. A complete low-resolution spectrum from 12 to $150 \mathrm{amu} / \mathrm{q}$ can be acquired in $12 \times 30 \mathrm{~s}$ $=600 \mathrm{~s}$. Several $20 \mathrm{~s}$ spectra with the same settings will then be recorded either in sequence or cyclically and transferred one by one to the DPU. After statistical analysis, spectra recorded with identical settings will be added, compressed, and transmitted as full mass spectra. This procedure optimizes the scientific data return from the instrument.

\section{Results from FM (Laboratory Instrument) and FS (Space Instrument)}

Pre-launch calibration: Both FM and FS have been calibrated in the CASYMIR facility (CAlibration SYstem for the Mass spectrometer Instrument ROSINA; 


\section{LEDA512 Tests with MCP + slits / File A112340.dat / Sept 241999 \\ Detail around anode 256 part A \\ MCP Front $=-1400 \mathrm{~V}$ Back $=-300 \mathrm{~V} / \mathrm{T}$ integ $=150 / \mathrm{Nr}$. Accum $=2$}

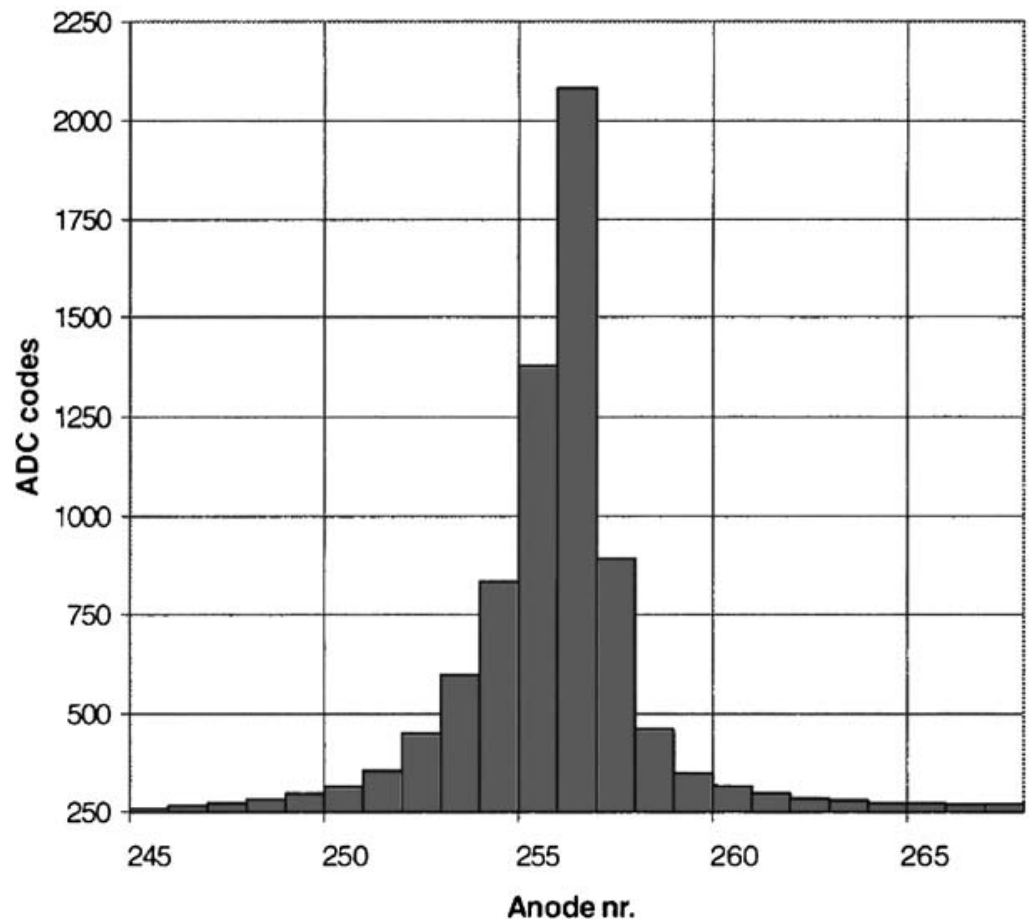

Figure 9. Example of the imaging detector signal for a $10 \mu$ wide ion beam impinging on the MCP.

Westermann et al., 2001) which provides neutral gas beams. Initial tests were conducted on the imaging detector to ascertain its resolution. These tests were achieved by sending an ion beam on a $10 \mu \mathrm{m}$ wide slit positioned just in front of the MCP entrance plate recording the readings from the LEDA electronics. An example of the results is given in Figure 9, which shows that the resolution achieved with the imaging detector is in accordance with the specification.

Static calibrations were done with $\mathrm{CO}_{2}, \mathrm{Ne}$, and $\mathrm{Xe}$ and yielded specific sensitivities of approximately $10^{-2}$ to $10^{-3} \mathrm{mbar}^{-1}$. These calibrations demonstrated that the instrument has a mass resolution of up to 3100 at $1 \%$ peak height and covers a dynamic range of up to ten orders of magnitude. Figure 10 shows a high resolution mass spectrum at $2 \times 10^{-7}$ mbar residual gas pressure taken with the CEM detector $(m / \Delta m=1900$ at $1 \%$ peak height $)$.

Performance in flight : After the launch of Rosetta in March 2004, several background and calibration measurements were done during commissioning phases. A low resolution mass spectrum over the full mass range is shown in Figure 11. 


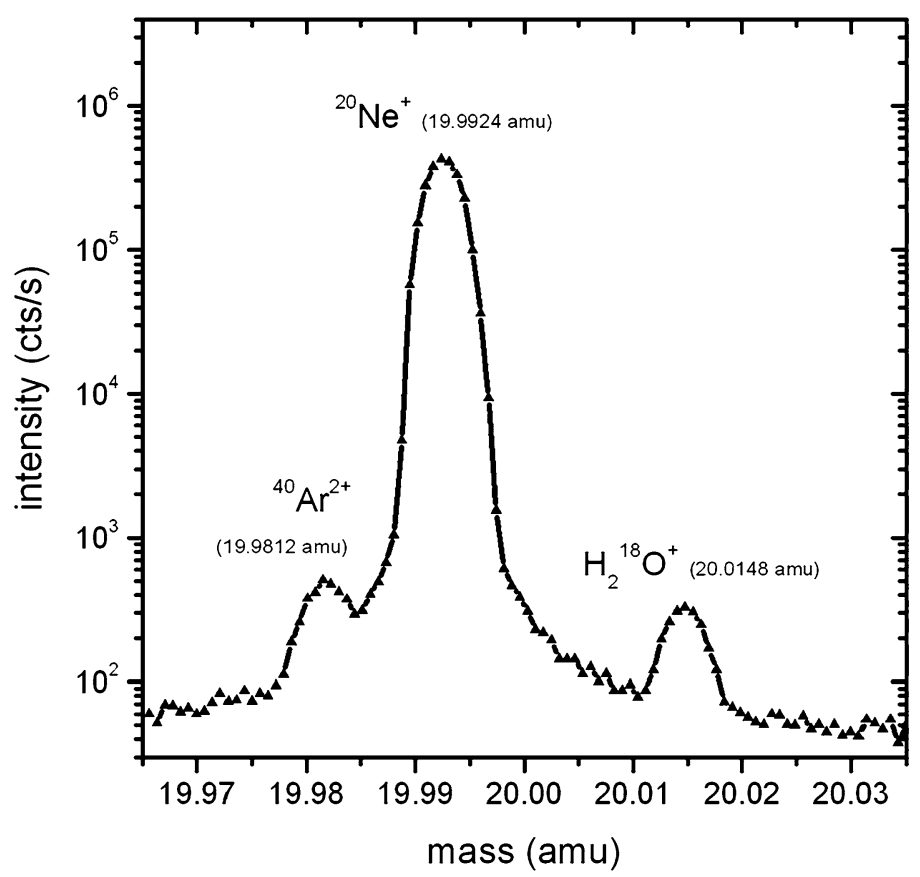

Figure 10. A high resolution mass spectrum of a mixture of noble gases at mass $20 \mathrm{amu}$ with the DFMS qualification model and a movable channeltron detector. Mass resolution is 2700 at the $1 \%$ peak height level.

Part of the high-resolution spectrum is given in Figure 12. Figure 13 shows an in-flight calibration spectrum of Xenon, taken in the low-resolution mode of the MCP detector.

The aforementioned measurements demonstrate that the DFMS sensor meets its requirements in flight. In particular, it has very high mass resolution, very high dynamic range, and good sensitivity.

\section{RTOF}

The reflectron time-of-flight (RTOF) mass spectrometer was designed for an extended mass range and high sensitivity to complete the instrument requirements of the ROSINA package. TOF instruments have the inherent advantage that entire mass spectra are recorded at once, without the need of scanning the masses by varying some particular instrument parameter like the magnetic field. A storage ion source stores the continuously produced ions until their extraction into the TOF section. With high transmission into the TOF section and a sensitive detector, it is possible to record a very large fraction ( $>60 \%$ in the case of RTOF) of all ions produced in the ion source. These factors contribute to the overwhelming 


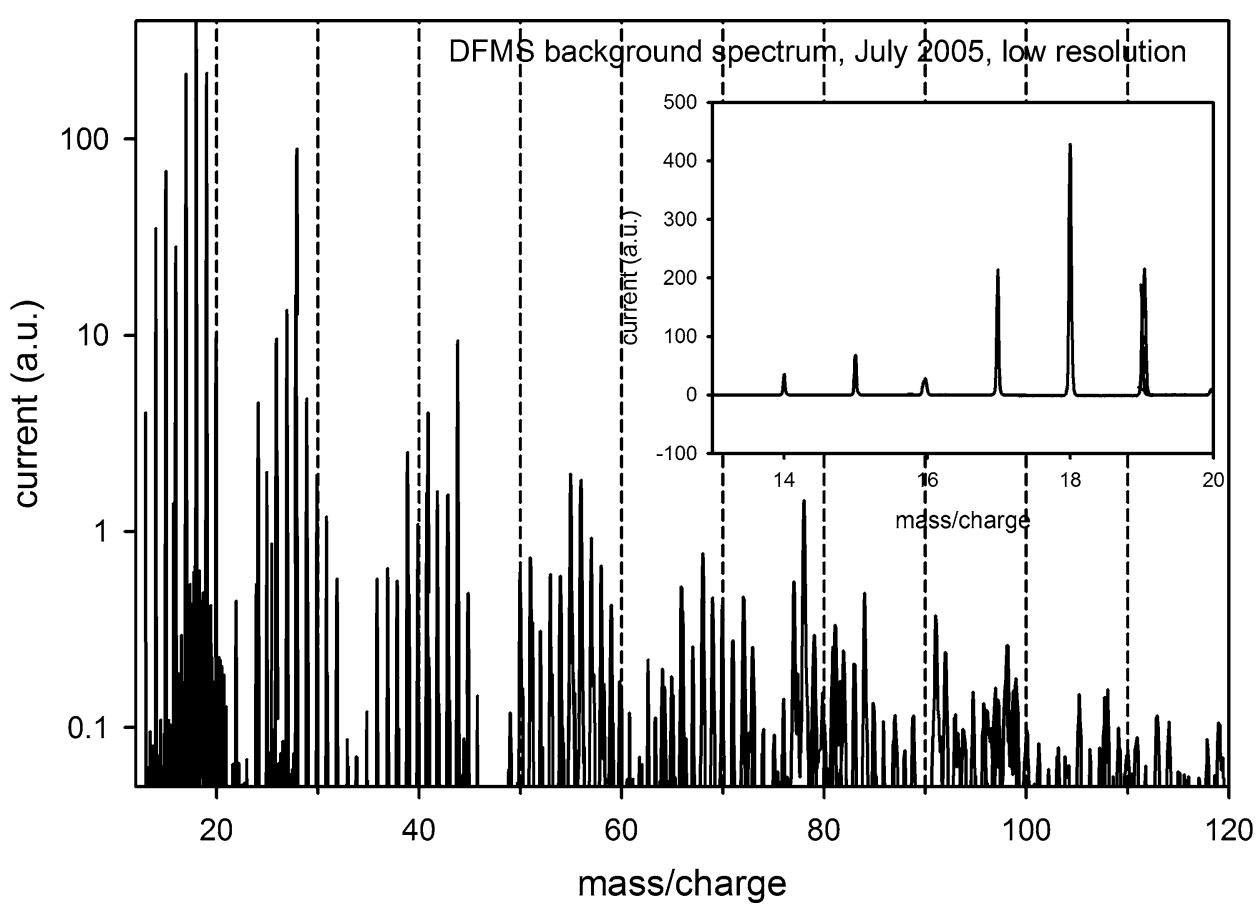

Figure 11. Low resolution background mass spectrum from space at a total pressure of $4 \times 10^{-11}$ mbar. Integration time was $20 \mathrm{~s}$ per mass group. The inset shows the mass range 13-20 amu/e in a linear scale. There is a mass peak on almost all integer mass numbers between 13 and $120 \mathrm{amu} / \mathrm{e}$.

sensitivity of TOF instruments. Another reason to use TOF instruments in space science is their simple mechanical design (their performance depends on fast electronics rather than on mechanical tolerances) and easy operation. A RTOF-type instrument was successfully flown on the GIOTTO mission to measure atoms and molecules ejected from a surface during impact of fast cometary dust particles (Kissel et al., 1986).

Figure 14 shows the principal ion-optical elements of the RTOF sensor together with calculated ion-optical trajectories. A time-of-flight spectrometer operates by simultaneous extraction of all ions from the ionization region into a drift tube in form of short ion packets. The temporal spread of such an ion packet is compressed from about $800 \mathrm{~ns}$ at the exit of the ionization region to about $3 \mathrm{~ns}$ at the first time focus plane (for mass $=28 \mathrm{amu} / \mathrm{e}$ ) at the beginning of the drift section. These very short ion packets then pass the first leg of the drift section, the grid-free reflectron, and the second leg of the drift section until they arrive at the detector. Because different $m / q$ packets drift with different velocities, the length of the drift section determines the temporal separation of ion packets of different $m / q$ when arriving at the detector. If properly matched to the field-free drift section the ion mirror, i.e. the reflectron, establishes the isochronity of the ion-optical system, which 

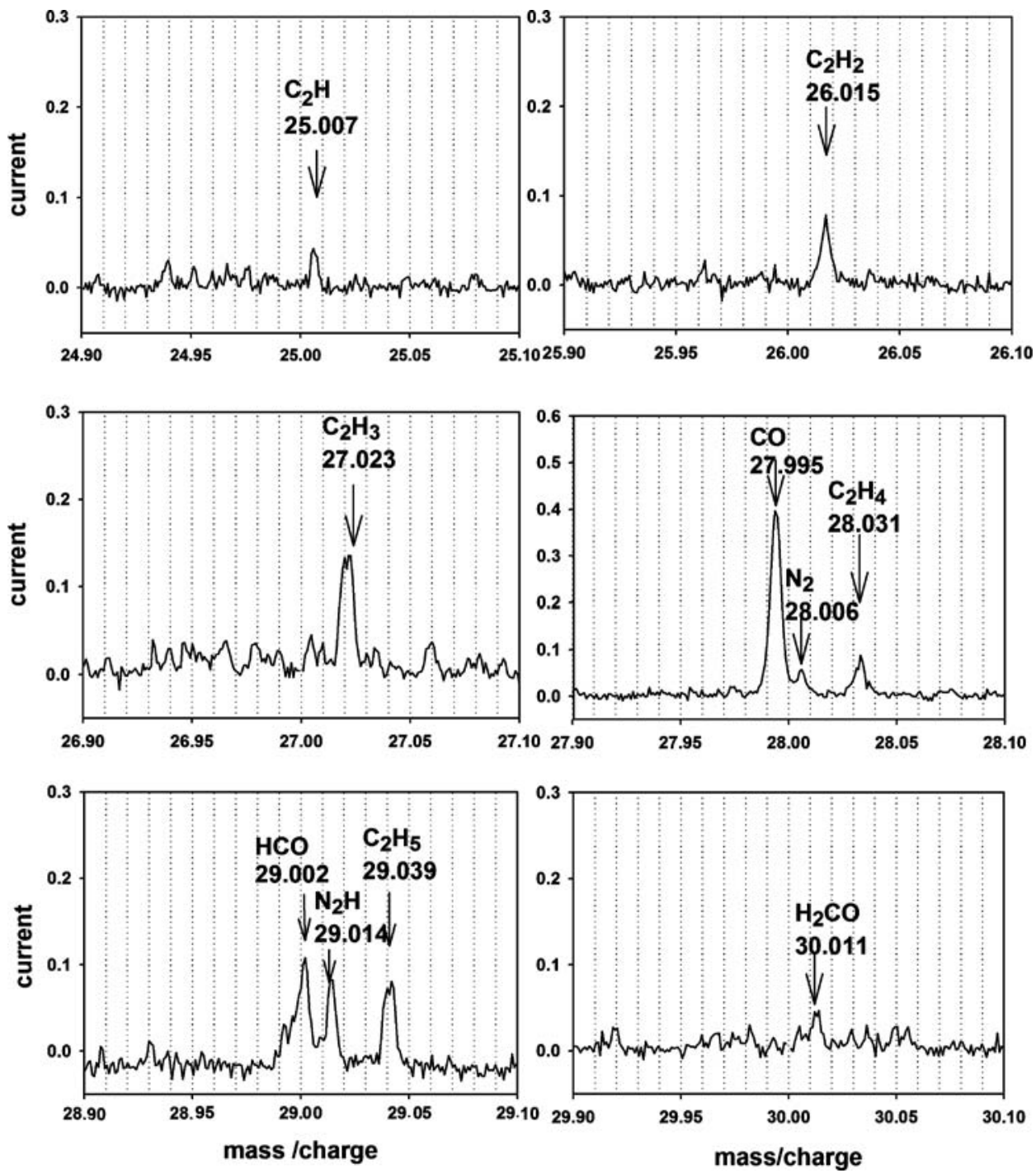

Figure 12. Part of a high resolution background mass spectrum from space at a total pressure of $4 \times 10^{-11}$ mbar. Integration time was $20 \mathrm{~s}$ per mass. The triplets at mass/charge 28 and $29 \mathrm{amu} / \mathrm{e}$ can be separated easily.

means that the flight time of ions is independent of their initial energy. The mass resolution is determined by the total drift time and the temporal spread of the ion packets at the location of the detector, which is placed at the last time focus. Unlike other types of mass spectrometers, TOF spectrometers have no limit to the mass range. In practice, the mass range is limited by the size of the signal accumulation memory. 


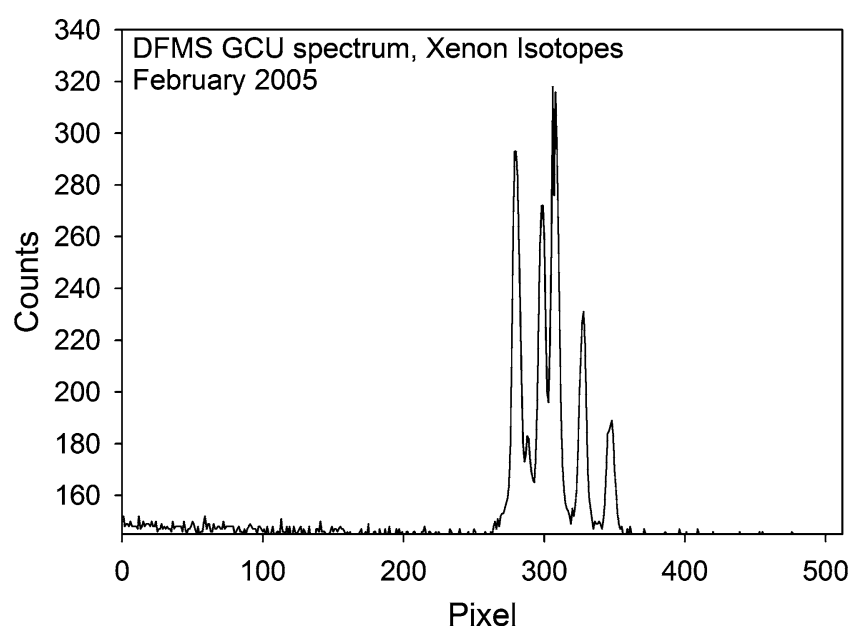

Figure 13. Part of an in-flight calibration mass spectrum from space at a total pressure of $5 \times$ $10^{-7}$ mbar test gas. Shown are the Xenon isotopes in low resolution. Integration time was $20 \mathrm{~s}$.

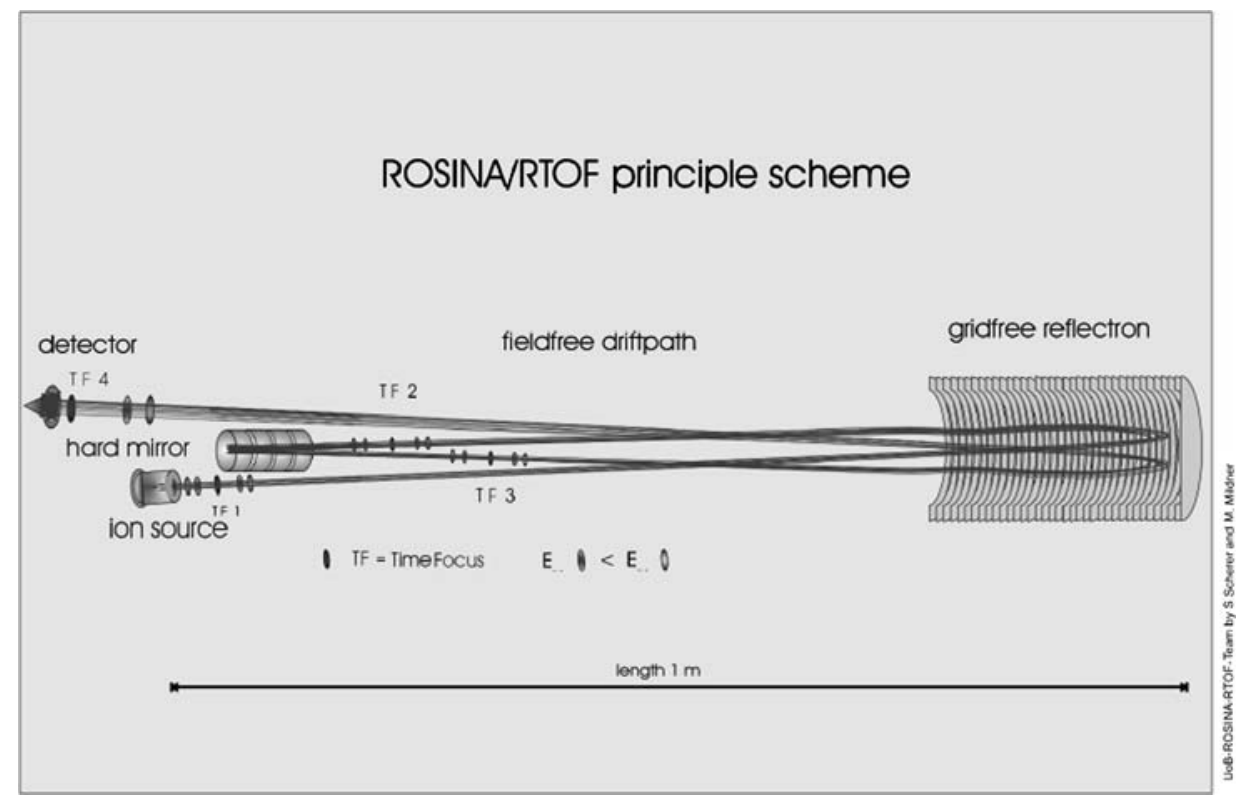

Figure 14. Ion optical principle of RTOF.

\section{Ion Optics}

The RTOF sensor consists of five ion-optical components: the ion sources, the drift tube, the reflectron, the hard mirror, and the detectors. The sensor includes two almost independent mass spectrometers in one common structure. The spectrometers share the reflectron and the hard mirror; however, the ion sources, the detectors, 


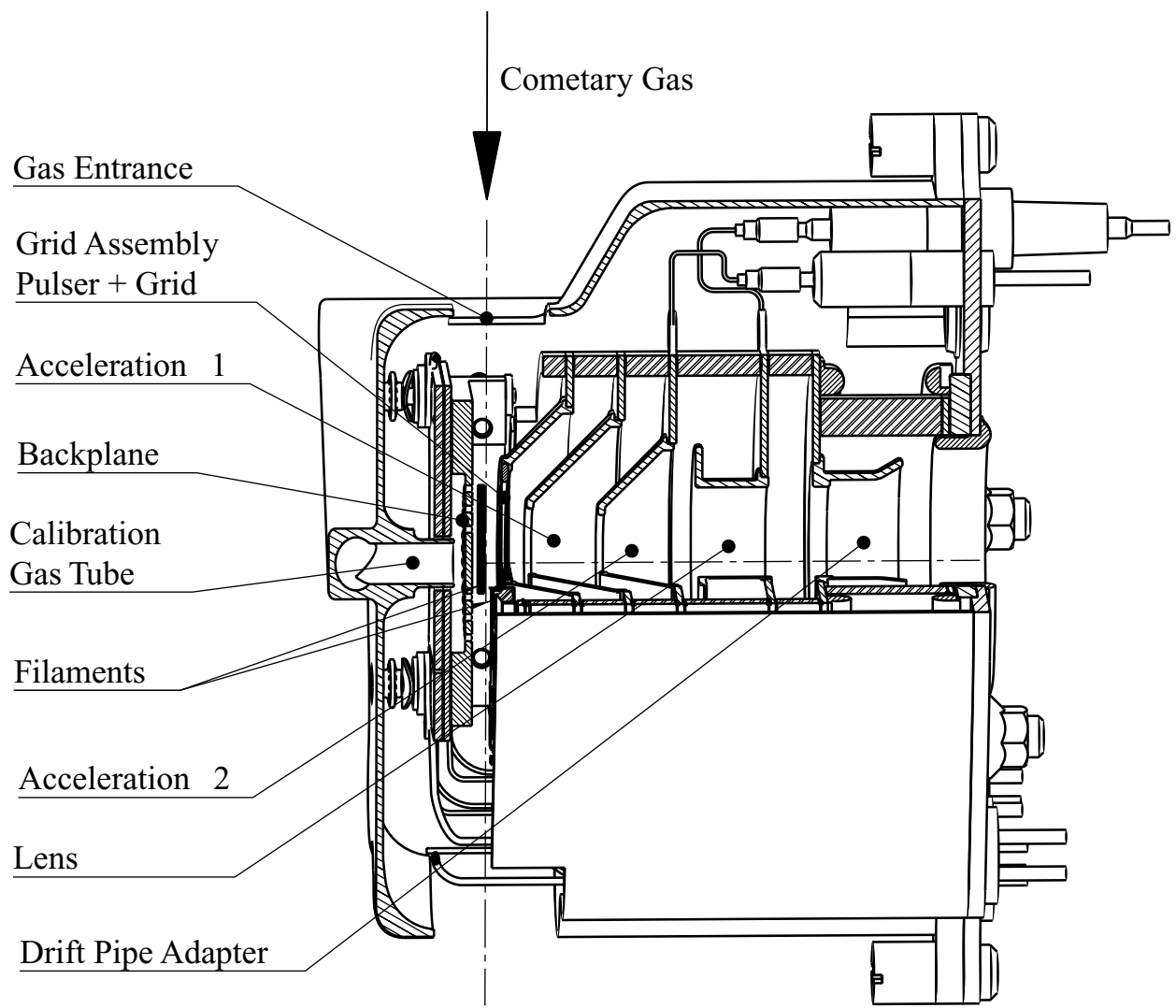

Figure 15. Schematic of the RTOF electron impact storage ion source,

and the data acquisition systems are separate. For the analysis of cometary neutral particles, there is the electron impact storage ion source with associated ion-optical elements and data acquisition (the storage-channel), and for the analysis of cometary ions there is the orthogonal extraction ion source with associated ion-optical elements and data acquisition (the ortho-channel). Both channels are optimized for their distinct purpose but have the feasibility to perform the other measurement as well. This configuration guarantees high reliability through almost complete redundancy.

Electron impact storage ion source: To achieve high sensitivity it is necessary to produce ions continuously, to store the created ions for a certain time, and to extract them at regular intervals into the TOF analyzer. The electron impact storage ion source, which is shown in Figure 15, performs these functions. The ion source is rotationally symmetric with the exception of the filament-repeller assemblies. The section plane shown in Figure 15 is representative for the three-dimensional model. 
For redundancy reasons, the ion source contains two filament assemblies, one on each side of the ionization region. Only one filament is active at a time, and it emits electrons that are accelerated to energies of typically $70 \mathrm{eV}$ or more. The electrons are guided through the ionization region using the two repeller electrodes. The inactive filament-repeller assembly located on the opposite side of the ionization region is used as electron trap to monitor the electron emission. The electron emission is regulated to assure a constant and reproducible ion production.

The electron beam continuously ionizes gas atoms in the ionization region, which is the volume between the backplane and the extraction grid (see Figure 15). The created ions are trapped in the potential depression resulting from the space charge of the electron beam. For a nominal ion extraction rate of $10 \mathrm{kHz}$, the trapped ions have to be stored for $100 \mu$ s before they are extracted by a highvoltage pulse that lasts for $1 \mu \mathrm{s}$. The applied extraction voltage is about $-320 \mathrm{~V}$. This corresponds to an electrical field strength of $E_{\mathrm{s}}=160 \mathrm{~V} / \mathrm{mm}$ in the ionization region (the distance between the backplane and the extraction grid) of $2 \mathrm{~mm}$ length as shown in Figure 15. The final energy of the extracted ions is obtained after passing two acceleration electrodes, acc 1 and acc2. An additional electrostatic lens located after the acceleration electrodes forms a parallel ion beam of diameter $\approx 5 \mathrm{~mm}$ at the ion source exit. For the nominal total ion energy of $3.0 \mathrm{keV}$, this extraction voltage results in a maximal energy spread of $10 \%$ for the ions.

The extracted ions are compressed to ion packets of several nanosecond duration at the time focus plane located approximately $5 \mathrm{~cm}$ after the ion source. By a suitable choice of the acceleration potentials second-order focusing at the first time focus plane is achieved, similar to the two-field ion source by Wiley and McLaren (1995). The temporal spread of the ion packet at the first time focus plane is then mostly governed by the turn-around time in the extraction region. The turn-around time is the time necessary to reverse the direction of an ion with its initial velocity (typical $0.1 \mathrm{eV}$ ) directed against the extraction direction of the ions by the extraction field in the source. The mass resolution of the RTOF sensor is mainly determined by temporal spread of the ion packets at the first time focus, thus given by the performance of the ion sources.

Orthogonal extraction ion source: The concept of the orthogonal extraction ion source was initially introduced for cluster ion measurements to provide an improvement to the limited resolution of conventional TOF instruments of the Wiley-McLaren type. The orthogonal extraction ion source allows for easy coupling of a TOF-MS with a wide range of external continuous or pulsed ion sources. In the case of the RTOF sensor, the orthogonal extraction ion source is optimized for the measurement of the cometary ions.

The orthogonal extraction ion source uses off-axis created ions. These ions are either coming from an external ion source (the comet) or using ions formed by electron impact ionization in an off-axis electron impact ionization assembly. The orthogonal extraction ion source is shown in the schematic illustration of Figure 16. 


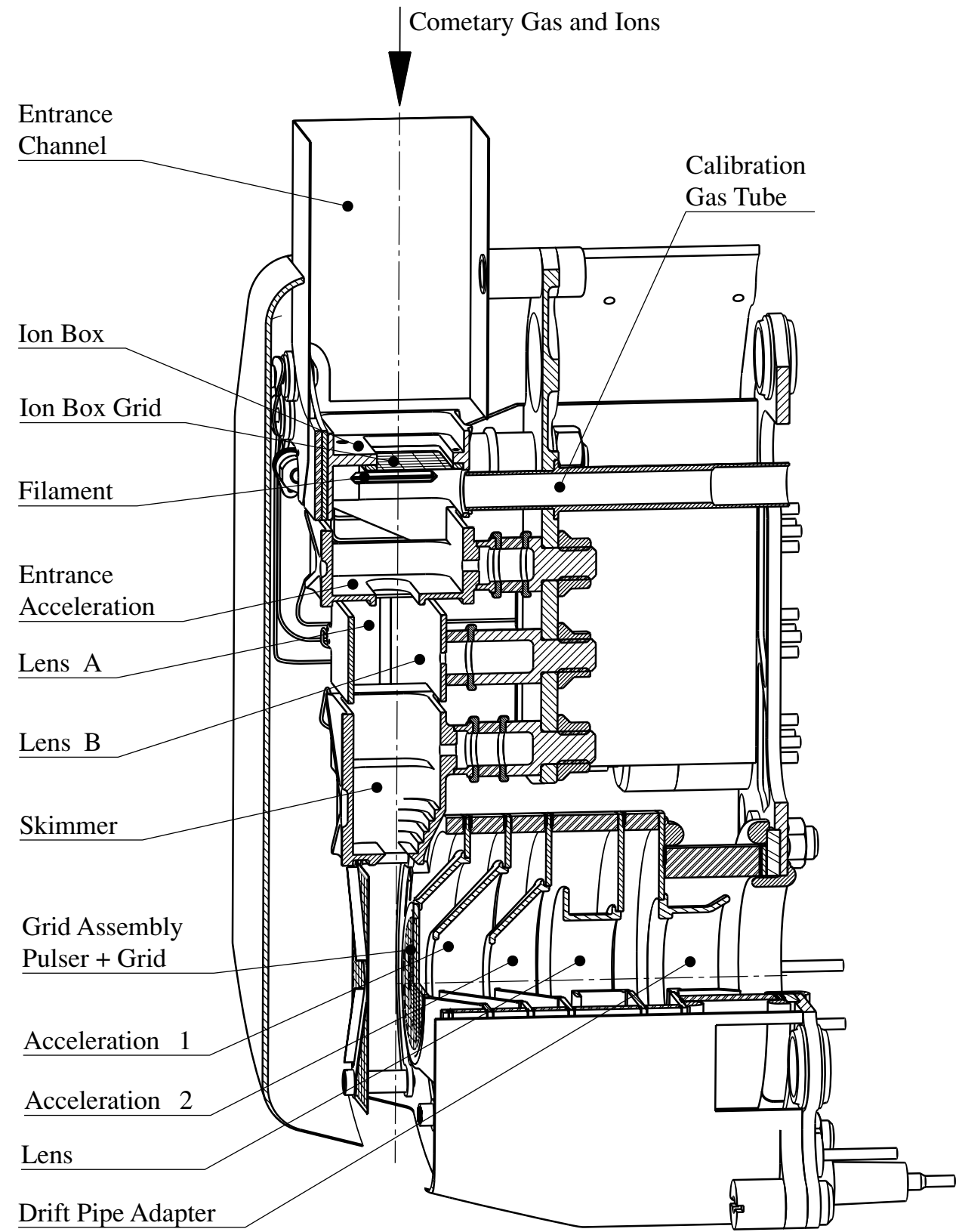

Figure 16. Schematic of the RTOF orthogonal extraction ion source.

These ions propagate orthogonally to the principal ion-optical axis of the TOF system with an initial energy of about $10 \mathrm{eV}$. When passing through the extraction region of the orthogonal extraction ion source, part of these ions are extracted by a fast high-voltage pulse on the extraction grid and are further accelerated onto 
the drift path of the TOF system. The small duty cycle resulting from pulsing ions out of a continuous ion beam is one of the major drawbacks of the orthogonal extraction ion source. The final energy of the extracted ions in the drift section again is $3.0 \mathrm{keV}$. The energy is achieved using a two-stage acceleration region allowing for second-order focusing at the first time focus plane.

The ion source consists of a rotational symmetric ion extraction and acceleration section and the off-axis ionization assembly mounted perpendicularly to the former (see Figure 16). The extraction and acceleration section of the orthogonal extraction ion source are the same as in the electron impact storage ion source. The filament and trap assemblies are planar symmetric in the plane of the drawing. Cometary ions are pulled into the entrance system of the off-axis ionization assembly by an external attraction grid and their energy is adjusted to be about $10 \mathrm{eV}$ by suitable acceleration or deceleration. The entrance section also has a filament assembly to create ions from in-flowing gas by electron impact ionization. The ions are accelerated to form a continuous ion beam orthogonal to the principal ion optical axis of the TOF system. The skimmer arrangement minimizes the velocity components in and against the direction of the principal ion-optical axis of the TOF system. Therefore, the turnaround time, which is the limiting factor for the mass resolution of the system, is largely reduced, resulting in a higher mass resolution of the ion channel than the gas channel.

Reflectron: The reflectron is the ion optical element of the RTOF sensor necessary to achieve the desired mass resolution. Basically, the reflectron is an ion-optical mirror at the end of a field-free drift path to redirect an incoming ion beam by an appropriate choice of repelling electrostatic fields. Thus, the field-free drift path is used twice and therefore the flight path is doubled, maintaining the overall geometrical dimensions of the RTOF sensor. The technical requirements for an application in spaceflight made it necessary to come up with a completely novel reflectron design, which is shown in a schematic illustration in Figure 17a and discussed in more detail in Scherer et al. (2005).

Because of the initial energy distribution of the ions, the temporal width of an ion packet will increase after the first time focus with increasing distance when moving along the field-free drift path. Since ions of higher energy have a shorter TOF, there is a negative time-of-flight dispersion. However, in the ion mirror, ions with a higher energy penetrate deeper into the repelling field before returning than do lower energetic ions. Consequently, the more energetic ions have a longer time of flight through the reflectron than the less energetic ones, resulting in a positive time-of-flight dispersion. By careful design of the electric fields of the ion mirror, the positive TOF dispersion compensates the negative time-of-flight dispersion on the field-free drift path over a wide energy range. This concept of time-focusing was first introduced by Mamyrin et al. (1973). The time-focusing property of the ion mirror for a given drift path length is often referred to as isochronous operation, since the flight time does not depend on the energy of the ions. The energy range of 
(a)
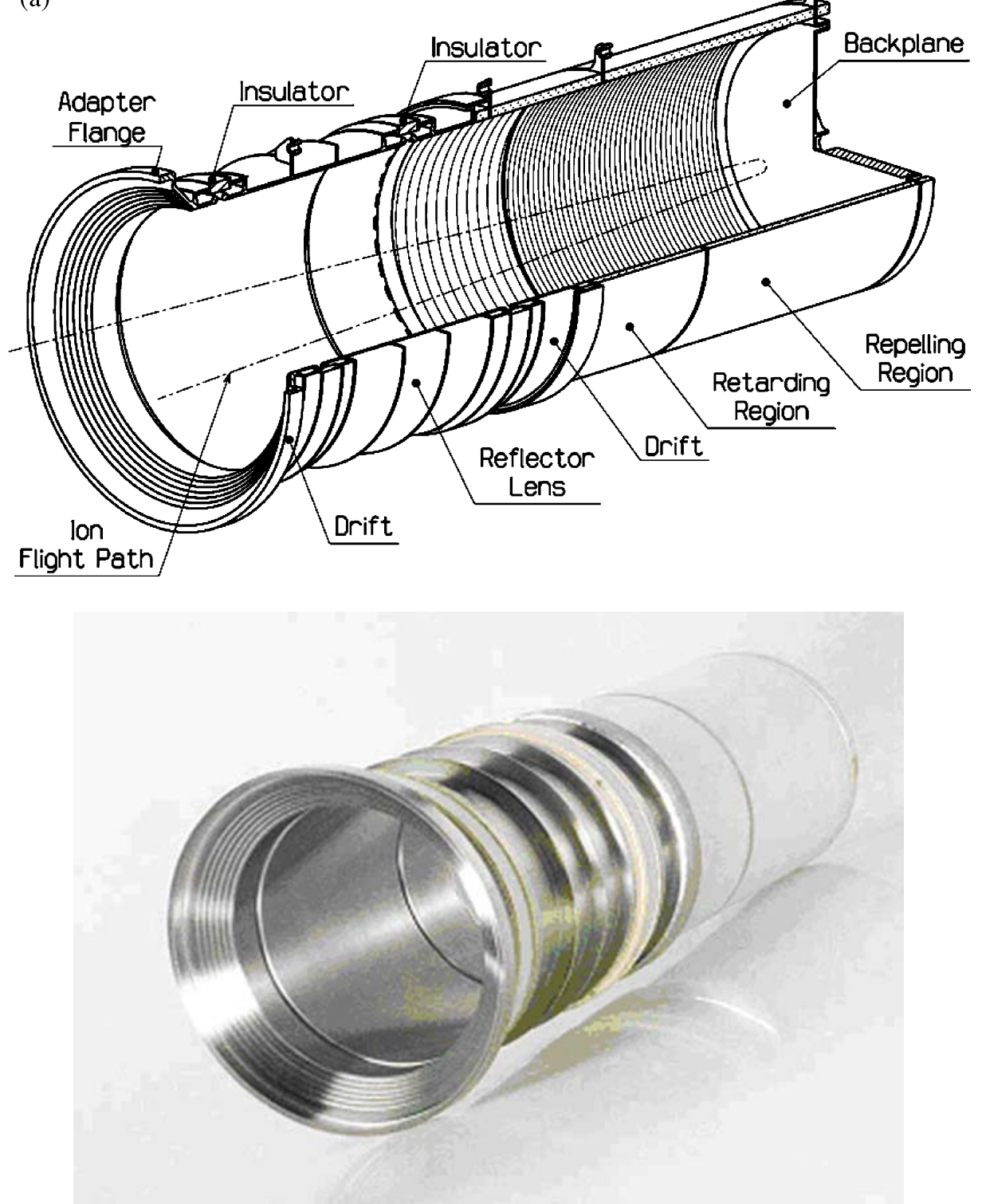

Figure 17. The integrated reflectron consists of an electrostatic lens in the entrance area and two following resistive helix segments, which generate the retarding and repelling potentials. The ions loose about two-thirds of their energy in the short retarding region and turn around close to the solid backplane. (a) Schematic of the reflectron and (b) the EQM integrated reflectron.

isochronous operation is $\pm 10 \%$ of the nominal energy for this design. Therefore, ions with a specific energy distribution and the same $m / q$ ratio will reach the detector plane simultaneously. The reflectron generates an image of the first time 
focus after the ion source to a time focus at the detector location with reversed velocity vectors for the ions.

The modification from a discrete two-stage reflectron to a grid-free reflectron is made by simply omitting the grids. Hence, the mechanical design of the reflectron is made easier and avoids transmission losses due to the limited ion-optical transparency of the grids, which is significantly lower than the geometrical transparency. However, the simple electrical field configuration with parallel equipotential lines changes into curved equipotential lines by superposition of the different potentials applied to form the retarding and repelling electrical field. The curved potential contour lines geometrically influence the passing ion trajectories. Therefore, a grid-free reflectron also has geometrical focusing or defocusing properties. Due to the positive voltages in the reflectron with respect to the drift path, the entrance region acts like the first half of a positive electrostatic lens. A grid-free reflectron always shows lens effects for ions not traveling along the ion optical axis. An ion beam will diverge in the entrance of a reflectron as it does in the entrance of a positive Einzel lens. To reduce the positive lens effect of the reflectron itself, a negative lens, i.e., the reflectron lens, is used at the reflectron entrance. The reflectron lens allows to optimize the geometrical focusing with the potential on the reflectron lens while, independently, the time focusing is optimized with the reflectron potentials. Additionally, the return angle of the reflected ion trajectories with regard to the ion optical axis of the system can be adjusted by the reflectron lens. The practical application of this feature is demonstrated for the different operation modes of the RTOF sensor (Hohl et al., 1999; Scherer et al., 2005). For a grid-free reflectron with an integrated electrostatic lens at the entrance, the angle of incidence is not equal to the angle of emergence. This property is unlike a conventional ion mirror with grids.

The electric fields for a reflectron are usually established by a set of rings connected to a resistive voltage divider. For RTOF, a novel approach for generating the retarding and repelling electrical fields was used where the voltage divider is an integral part of the reflectron (this approach is called an integrated reflectron). The voltages and thus the electrical fields are defined by a voltage drop over a resistor in the form of a helix applied to the inner surface of a ceramic tube with an inner diameter of $78 \mathrm{~mm}$ (see Figure 17a). The potential drop is constant along the helix and compensates exactly for the helix pitch. Therefore, the integrated reflectron shows no electrical fringe field zone in close proximity to the cylindrical boundary given by the mechanical structure (Scherer et al., 2005). An ideal electrical field for the grid-free reflectron is generated in the entire inner volume of the structure. The total resistance over the helix is designed to be about $10^{9} \Omega$. The resistance has to be high to keep the power consumption of the HV supply low but also it has to be low enough that absorbed charges cannot change the potential distribution in a noticeable way. The minimum resistance is determined from the maximum ion current of about $1 \mathrm{nA}$ extracted from the ion source and the required adjustment accuracy for the reflectron voltages of about $1 \mathrm{~V}$. 
The resistor helix is painted in a specially developed procedure at the inner surface of a ceramic tube, and afterward is subjected to a sintering process. Manufacturing and processing of the integrated reflectron was performed at GVE/EMPA in Zürich, Switzerland. A photograph of the realized integrated reflectron is reproduced in Figure 17b. The helix consists of two segments, where the length of the retarding segment is half the length of the repelling segment, but the voltage drop over the retarding segment is two times the value for the repelling segment, according to the second-order focus conditions. The voltage drop over the reflectron is controlled at three points.

Furthermore, the ceramic structure of the integrated reflectron acts simultaneously as the UHV enclosure for the RTOF sensor and is an integral part of the overall mechanical sensor structure. The ratio of the ion optically usable inner diameter $(78 \mathrm{~mm})$ and the mechanical outer diameter $(80 \mathrm{~mm})$ is minimized. This results in a 50\% weight saving compared to the classical ring stack reflectron, which requires an additional vacuum housing and high voltage feedthroughs. Following thermal conditioning, the pump valve was closed and the vacuum pressure inside the integrated reflectron stayed in the high $10^{-8}$ mbar range for more than 1 week. Actually, the titanium elements of the integrated reflectron acted as an active getter surface and the pressure inside the integrated reflectron even decreased further with time. Successfully passing the vacuum-keeping test was a critical milestone in the RTOF development, since the sensor had to be launched into space fully evacuated to UHV levels to avoid contamination by atmospheric gases or rocket propellants.

Hard mirror: The hard mirror represents an additional ion mirror in the RTOF system (Hohl et al., 1999) and is shown schematically in Figure 18. The hard mirror increases the flight path (and therefore the mass resolution) in the RTOF system by almost a factor of two by introducing an additional reflection while maintaining the initial time spread of the ion packets generated in the ion source. The hard mirror consists of cylindrical tubes and a backplane whose shape has been optimized by ion-optical simulations. Restricted by the limited space between ion source and detector (see Figure 14), the hard mirror has to fit geometrically in the TOF system without substantially increasing the distance between ion source and detector to keep the angle between the incoming ion trajectory and the outgoing trajectory in the reflectron as small as possible. The reflecting region is short compared to the grid-free reflectron and thus performs a "hard" reflection, which means the penetration depths of ions of different energies are almost the same. The time focus of the hard mirror is chosen to be close to its exit plane, since the hard mirror has limited time-focusing capabilities because of the hard reflection. Similar to the gridfree reflectron described earlier, the hard mirror contains a negative electrostatic lens at the entrance to shape the ion trajectories spatially. Subsequently, the cylindrical electrodes following the hard mirror lens, in conjunction with the backplane, allow the adjustment of the retarding and repelling electrical fields. 


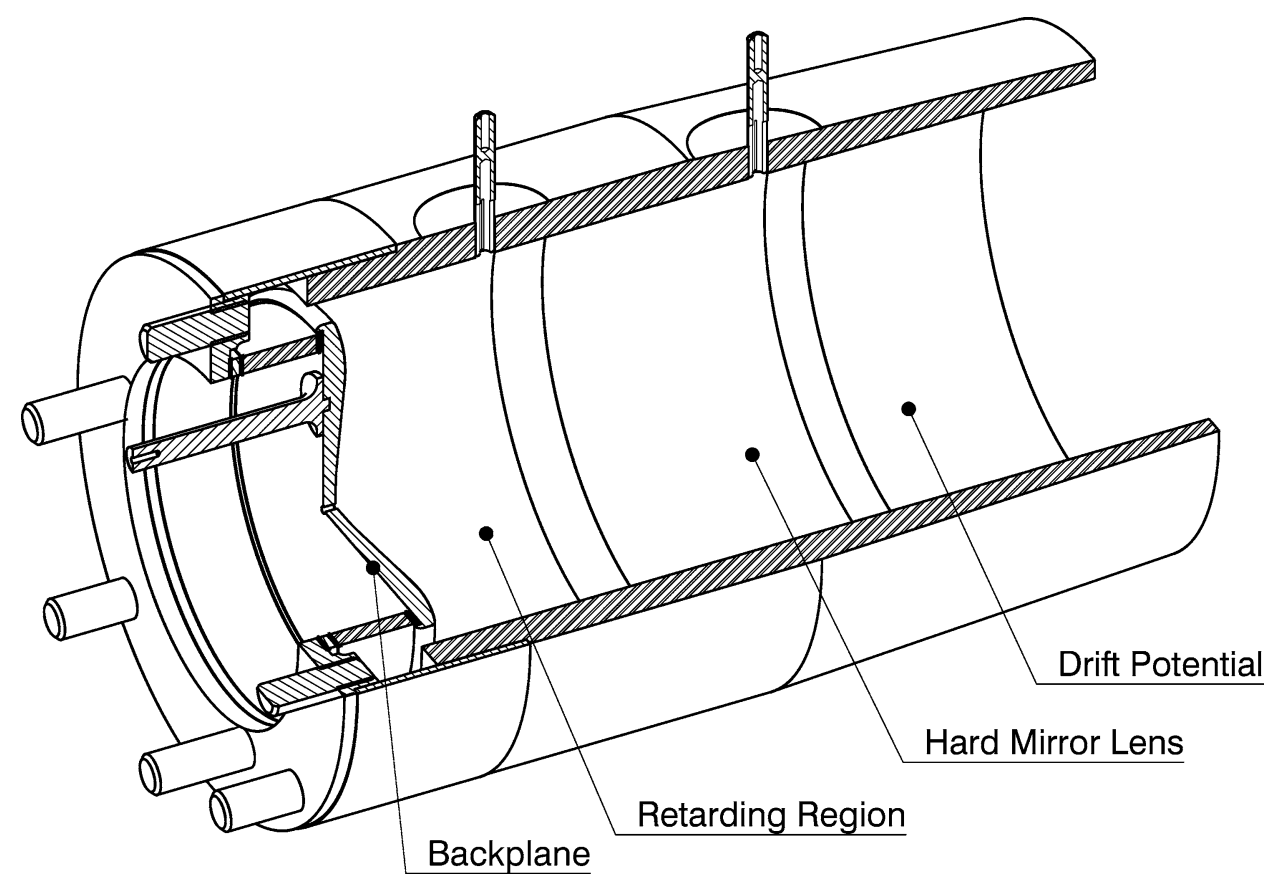

Figure 18. The hard mirror structure is based on a ceramic tube body with an inner diameter of $36 \mathrm{~mm}$. Three conductive silver ring electrodes are applied on the inner surface of the ceramic body, with a sophisticated shaped backplane defining the repelling electrical field. The hard mirror contains a conductive outer surface connected to the first inner ring electrode on drift potential to avoid electrical field disturbances affecting ion trajectories passing close by the hard mirror.

The hard mirror offers the unique opportunity to suppress selected mass lines by applying a pulsed defocusing voltage to the backplane electrode (see Figure 18). The electric field during the applied "blank-pulse" results in a strong geometrical defocusing of ions traveling not along the rotational symmetry axis. Thus, these ions will be lost by scattering inside the drift tube structure and will not reach the detector. Due to the short image length of the hard mirror, the longitudinal separation of iso-mass ion packets in the hard mirror itself is small compared to the hard mirror length, and allows for moderate blank-pulse amplitudes to exclude individual mass lines. The pulse slope requirements are less stringent compared to the extraction pulse.

The hard mirror structure for the flight model is based on a ceramic tube body with an inner diameter of $36 \mathrm{~mm}$. Three conductive silver ring electrodes are applied on the inner surface of the ceramic body with a sophisticated shaped backplane defining the repelling potential surface. The ion trajectories starting at the ion sources pass in the immediate vicinity of the hard mirror and thus demand an appropriate shielding of the electrical fields of the hard mirror to the outside, the field-free drift region. For this reason, the hard mirror has a conductive coating 
on the outer surface connected to drift potential and an extended entrance ring electrode with the same potential as the drift section.

Detector: Detecting single ions as well as ion bunches with up to $10^{5}$ ions arriving within nanoseconds time requires a detector with high detection efficiency. Furthermore, the detector has to have the ability to linearly amplify the incoming particles over a wide dynamic range. In order to minimize the time spread of the ion bunches registered on the detector, sufficiently fast detectors with an internal time response for single-ion events of less than $1 \mathrm{~ns}$ have to be used. A narrow time width not only improves the mass resolution but also increases the peak amplitude and therefore improves the signal-to-noise ratio. The geometry of the ion-optical system of RTOF limits the diameter of the ion beam to $12 \mathrm{~mm}$ on the detector. For mass saving reasons, the active area of the detector is therefore only $18 \mathrm{~mm}$. Microchannel plates (MCPs) of imaging quality have been selected for registering the ions. Micro-sphere plates were investigated as well but turned out not to be stable enough over the projected life time of the RTOF sensor.

The critical issue for a fast detector is the anode design, which has to ensure a $50 \Omega$ impedance matching when coupling of the electron pulse released from the channel plate into a standard transmission line with minimal signal reflections and distortions (Wurz and Gubler, 1994; Wurz and Gubler, 1996). The detector output is capacitively decoupled from the anode and thus allows the detector to float electrically. The transmission line yields directly into a SMA output connector to connect the signal line. The signal is routed through a semi-rigid cable (impedance $Z_{L}=50 \Omega$ ) and high-frequency tri-axial vacuum feed-through (rated $4 \mathrm{GHz}$ ) to the data acquisition system to minimize the noise pickup. The measured pulse width for a single-ion event of this detector including the signal routing is about 500 ps (Schletti et al., 2001; Siegmund et al., 2001). The detector contains passive electronic components to provide the various voltages and charge buffering needed to supply the two MCP stages in the detector. The detector can be operated in analog mode or in pulse counting mode.

Gas calibration unit: The GCU is used to inject a defined quantity of a known gas mixture $\left(\mathrm{He}, \mathrm{CO}_{2}\right.$, and $\mathrm{Kr}$ ) into either the storage source or the orthogonal source. By feeding a source with a known gas mixture (with well-known masses), the sensor parameters can be optimized, the detection efficiency can be calibrated, and the performance can be evaluated in flight.

For the two ion sources, two independent GCUs are implemented, which are controllable by remote commands. Both GCUs are accommodated in a common housing and mounted on the electronics box. Each GCU consists of a gas tank of $5 \mathrm{~cm}^{3}$ filled with about 5 bars of the calibration gas mixture, a high-pressure gauge, a valve, a low-pressure gauge (mini pirani) and a capillary tube with a standard CAJON vacuum connection at the GCU exit. From there the gas is routed with regular gas tubing into the ionization region of the ion sources. All subcomponents 
were fabricated very clean to avoid any gas contamination. Leakage rates for all components and mounted capillary tube with closed valve are $\leq 10^{-10} \mathrm{mbar} 1 / \mathrm{s}$. The controllable leak rate using the low-pressure gauge can be set between $10^{-3} \mathrm{mbar} 1 / \mathrm{s}$ and $10^{-1} \mathrm{mbar} \mathrm{l} / \mathrm{s}$. With this leak rate, the apparent pressure in the ionization region of ion sources is in the range from $10^{-9}$ to $10^{-6}$ mbar.

The GCU is separately powered by $\pm 5 \mathrm{~V} \mathrm{DC}$ and $+6 \mathrm{~V}$ (for valve heater) in both GCUs. The output pressure of the individual GCUs are controlled in a closed loop circuitry. A set value is used as control value (known from calibration) and a converted pressure value is detected via housekeeping every $200 \mathrm{~ms}$ by the data processing unit (DPU). The DPU software compares between the set value and measured value that leads to the ON/OFF command for the valve heating. This command is sent to the main controller (MC) that switches the selected valve heater ON or OFF.

\section{RTOF Operation Modes}

The RTOF flight instrument provides several operation modes to assure optimal scientific data return under diverse mission conditions. The fundamental modes are the storage channel and orthogonal channel modes, with their dedicated ion sources and their own optimized data acquisition system. The RTOF sensor on the Rosetta spacecraft has the following operational modes.

Storage channel mode: The storage channel mode is assigned to the electron impact storage ion source and analyzes initially neutral particles. During the storage period up to $10^{5}$ ions will be accumulated in the ion source and extracted by a high-voltage pulse into the TOF analyzer section. The Equivalent Time Sampling (ETS) data acquisition system, which is described below, records the detector signal proportional to the number of incoming ions.

Orthogonal channel mode: The orthogonal channel mode is performed with the orthogonal extraction ion source optimized to analyze cometary ions. The Equivalent Time Sampling Light (ETSL) data acquisition system, which is described below, counts the registered ions extracted from the orthogonal extraction ion source. Moreover, the orthogonal extraction ion source also has the ability to ionize incoming neutral particles with a filament assembly using electron impact ionization. Both ion sources could therefore be used to detect neutrals as well as ions.

Single- and triple-reflection mode: The single-reflection mode refers to the ion trajectories starting at the ion source, being one time reflected in the reflectron and the trajectories ending at the detector (see Figure 14). This mode produces a large instantaneous mass range with medium mass resolution.

In the triple-reflection mode, the ions leave the ion source, reverse their direction of motion for the first time in the reflectron, and experience a second reflection in the hard mirror. After a third reversal of their direction of motion in the reflectron, 
they hit the detector. The reflectron is used twice in this mode and the hard mirror is passed once (see Figure 14). This mode produces high mass resolution with a smaller instantaneous mass range.

Switching between the single- and triple-reflection mode is performed by changing the voltage of the reflectron lens. The single-reflection mode requires a typical reflectron lens voltage of about $-2500 \mathrm{~V}$ below the drift potential, whereas the triple-reflection mode operates with a reflectron lens voltage of $-4000 \mathrm{~V}$ below the drift potential. There is no mechanical tilt elements operated in flight nor are there electrical deflection plates, which could redirect the ion beam between the single and triple reflection mode. The storage and the ortho channel can be used simultaneously but they always must operate in the same mode (single- or triple-reflection) because of the commonly used reflectron structure and the differing voltage sets for the single- and triple-reflection mode.

Blank mode: The blank mode allows suppressing selected mass lines to prevent overloading of the detector in case of very intense mass lines (e.g., water ions) (Hohl et al., 1999). This mode is available only together with the triple-reflection mode, since the blank-pulse operation is performed with the hard mirror and requires synchronization of the extraction pulse with the hard mirror blank-pulse.

Calibration mode: The calibration mode allows the calibration of the detection efficiencies and the sensor optimization during flight by using the RTOF GCU. Upon DPU command, the GCU system releases a defined quantity of a calibration gas into the selected ion source. In addition, it has been foreseen to self-optimize the RTOF sensor by a software module of the DPU. To achieve optimal performance of the RTOF sensor the electrical parameters (e.g. voltages on the ion optical elements etc.) have to be fine-tuned carefully. In flight, the RTOF sensor is initially operated with a preset adjustment of the electrical parameters derived from the sensor calibrations. To achieve optimal performance in space (e.g. at a given sensor temperature), an automatic optimization algorithm will be used for fine-tuning the sensor parameters involving either the calibration system for the initial optimization or using cometary gas for routine optimization. The optimization process has to be performed autonomously on board the spacecraft by the ROSINA DPU due to a limited command and data transfer rate during the mission.

\section{Mechanical/Structure}

The RTOF sensor (see Figure 19a and b) consists of a primary structure containing all ion optical elements within an UHV enclosure, a cover opening mechanism, and a secondary structure, the electronics box, which houses the sensor electronics. The secondary structure also serves as mechanical support for the primary structure and for the in-flight calibration system. The primary structure of RTOF is made from titanium and ceramics. The sensor head, which is electrically at structure ground, is isolated electrically from the drift tube, which is at drift potential 

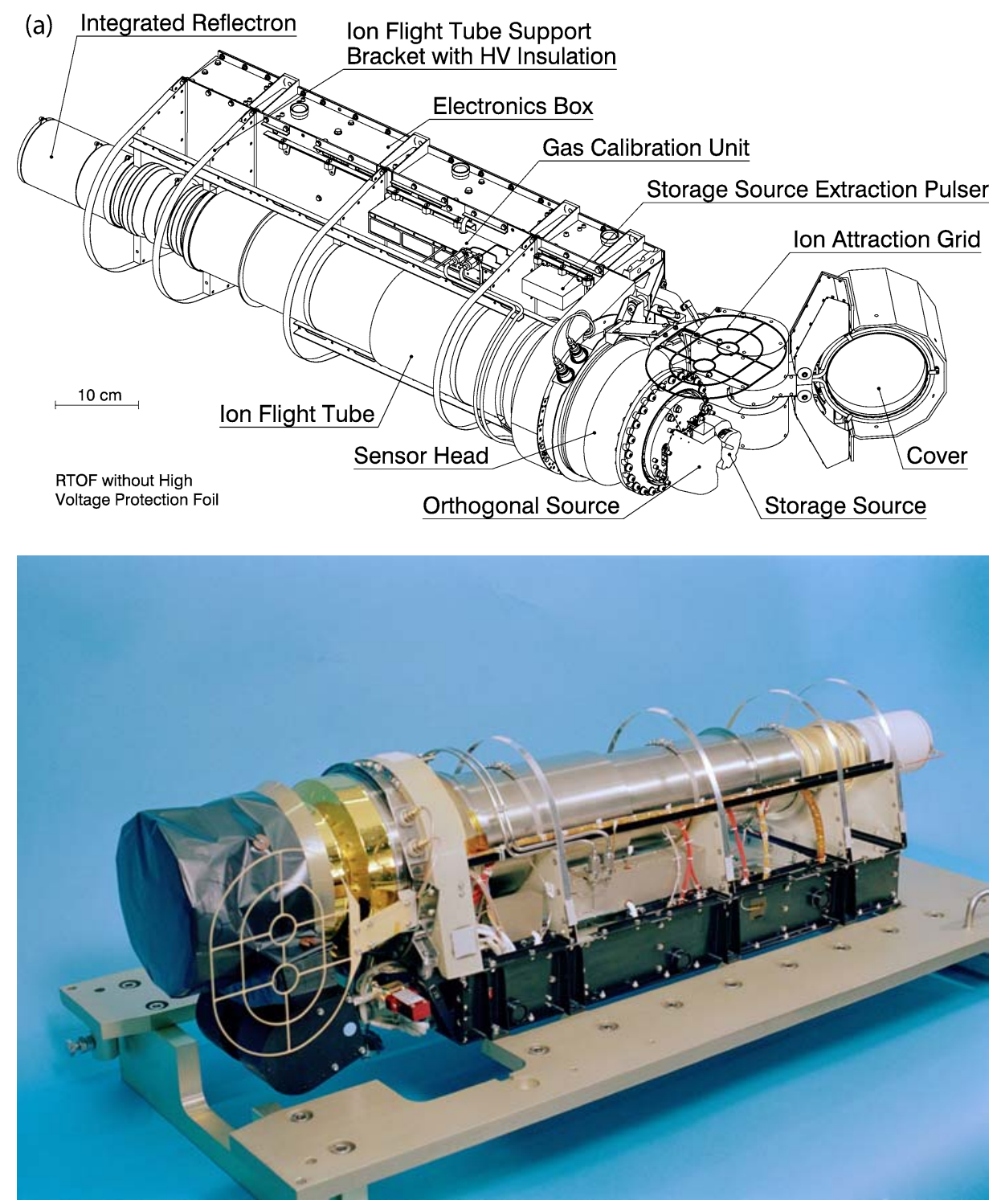

Figure 19. (a) Mechanical drawing of the RTOF flight sensor. (b) Picture of the RTOF flight spare with thermal hardware and ion attraction grid. Only the front part of RTOF is exposed to space, most of the instrument is inside the spacecraft.

(nominally $-3 \mathrm{kV}$ ), by a ceramic ring. A reclosable cover protects the sensor head with both ion sources. The mechanical structure of the field-free drift tube also serves as the UHV enclosure of the RTOF sensor. The potential applied to the drift tube defines the ion energy. The ceramic tube of the integrated reflectron is part of 
the entire RTOF vacuum enclosure. The sensor head, mounted at the opposite end of the drift tube, carries the electron impact storage ion source and the orthogonal extraction ion source with the respective detector as well as the hard mirror. The ion sources and the detectors are mounted in the sensor head at optimized tilt angles with respect to the ion optical axis of the system determined experimentally. The whole primary structure has been baked out to $150^{\circ} \mathrm{C}$ and the ion sources can be baked in flight up to $300^{\circ} \mathrm{C}$. The sensor is launched under UHV vacuum conditions and the cover was opened in space 2 months after launch to minimize contamination.

The secondary structure, the electronics box, is made from aluminum and houses the sensor electronics, which comprises eight electronics boards. The three highvoltage pulsers (two for the ion sources, one for the hard mirror) are mounted on the outside of the electronics box close to the sensor head. Also, the two RTOF GCU tanks are attached on the outside of the electronics box with gas tubing to the two ion sources.

\section{Electronics}

The entire electronics of the RTOF instrument consists of the following nine functional blocks:

- Main controller $(M C)$ : The MC handles the commands coming from the DPU and the data and housekeeping going to the DPU. It contains the following blocks:

- Motor mechanism for the cover.

- Backplane heater unit.

- GCU.

- Gas extraction pulser, ion extraction pulser, and hard mirror pulser.

- ETS and ETSL latch up disable.

- Filament emission.

- Differential serial interface to the DPU.

- Gateway switches for ETS, ETSL, and digital board.

- Housekeeping unit.

- Power switching unit.

- Equivalent time sampler (ETS): Data acquisition system for fast and nonrepetitive signal pulses for the storage channel.

- Equivalent time sampler light (ETSL): Data acquisition system for fast and repetitive pulses for the orthogonal channel.

- High voltage board \#1 (HV\#1): High-voltage supplies for ion sources, hard mirror, acceleration, lens, reflectron and drift tube.

- High voltage board \#2 (HV\#2): Supply for extraction pulsers, detectors, and hard mirror pulser voltages. 
- Low voltage power supply (LVPS): Supply for analog $\pm 5 \mathrm{~V}$, dig. $+5 \mathrm{~V},+8 \mathrm{~V}$, analog $\pm 15 \mathrm{~V},+24 \mathrm{~V},+40 \mathrm{~V},+70 \mathrm{~V}$.

- Digital board for power supplies: Backplane, entrance lens and entrance supplies, controller for the supplies, HK and MC, temperature sensors.

- Filament emission controller (FEC): The FEC regulates the emission current of the storage and the orthogonal source filaments, for the main and the redundant filament sets.

- Ion, gas and hard mirror pulser:

- The ion and gas pulsers perform the extraction with a negative pulse with a fast falling edge $\left(t_{\mathrm{f}}<5 \mathrm{~ns}\right)$ and a medium fast rising edge $\left(t_{\mathrm{r}} \sim 100 \mathrm{~ns}\right)$. The amplitude is programmable.

- The hard mirror pulser deflects charged particles before they hit the detector with a positive pulse from a positive hard mirror potential. Pulse width, delay from trigger and pulse amplitude are programmable.

\section{Equivalent Time Sampling (ETS) and the Equivalent Time Sampling Light (ETSL) System}

The ETS and the ETSL are the two data acquisition systems in the RTOF sensor. ETS is dedicated to the storage channel and ETSL to the orthogonal channel. Both data acquisition systems serve as time-to-digital converters (TDC); that is, whenever a signal exceeds a preset 3 bit programmable trigger level $(10-100 \mathrm{mV})$, the time of this event is stored in a memory. However, because the ion density is much higher in the storage source due to the longer duty cycle leading to multiple ions arriving at the same time on the detector, ETS is additionally capable of converting the signal height into a digital value thus serving as fast analogue-to-digital converter (ADC) paired with its TDC function.

Parameters like trigger level, TOF, etc. of the ETS and ETSL systems are serially commanded by the DPU. The data acquisition of events starts with a command. The systems generate then the periodic trigger for the source extraction pulsers. A 13 bit (maximum $217 \mu$ s) start delay time can be programmed prior to the start. Only after the elapsed time the data acquisition system starts accepting signals from the detector. This avoids overflow of the FIFO memory if there is a high event rate from the pulser crosstalk or for low masses. The maximum allowed time of flight $(\leq 217 \mu \mathrm{s})$ depends on the extraction frequency which can be selected between 1 and $10 \mathrm{kHz}$ and is further limited by the size of the data FIFO $(512 \times 18 \mathrm{bit})$ memory.

The ETS is a multiple ADC high-speed data acquisition system that is designed to record TOF spectra of fast and non-periodic pulses registered by the MCP detectors. Histograms of the spectra are recorded on the ETS. Sixteen high-speed, 8 bit low power ADC units are fired with a $1.65 \mathrm{~ns}$ tapped delay, after an input signal exceeds the trigger level of a high-speed comparator. It is delay is as small as $0.5 \mathrm{~ns}$ to minimize jitter, thus 16 data points cover $26.5 \mathrm{~ns}$. The delay between the sampled waveform and the first ADC start is less than $0.5 \mathrm{~ns}$. The analogue 
signal bandwidth is $\sim 1 \mathrm{GHz}$ to record waveforms with minimal signal distortion. The input is terminated to $50 \Omega$ and is AC coupled. The input is protected against voltages greater than $\pm 1.2 \mathrm{~V}$. An 8 bit conversion takes 2.5 clock cycles at $50 \mathrm{MHz}$. The ADC units are designed for asynchronous operation to save power. Each unit contains a sample and hold circuit with an aperture time of $0.35 \mathrm{~ns}$ and an ADC as well as the control logic and clock generation.

The 8 bit ADC data with an increment of $1.65 \mathrm{~ns}$ are accumulated to a 30 bit wide word for each TOF channel. The number of events per TOF channel is accumulated to 18 bits. The dead time between two trigger events, generated from an incoming waveform, is $\sim 133 \mathrm{~ns}$ in the standard mode.

In mass spectrometry mass peaks occur only at discrete flight times corresponding to integer mass numbers. Therefore, the dead time of the ADC has to be shorter than the difference in flight time for adjacent mass numbers. For the case that a time gap free sampling is required (e.g. to detect multiple charged ions), the system can be set into the "Delayed Time Sampling Mode", where the acquisition start delay value is not fixed, but increased automatically by $26.5 \mathrm{~ns}$ after each extraction.

For cases where a higher mass resolution is required (e.g. triplet at mass/ charge $28 \mathrm{amu} / \mathrm{e})$ the ETS can be commanded to a high resolution mode where the start of the ADC firing is delayed by $\mathrm{n}$ times $0.55 \mathrm{~ns}(n=0, \ldots, 2)$ relative to the trigger event. One spectrum is recorded during three $(3 \times 0.55 \mathrm{~ns}=1.65 \mathrm{~ns})$ extractions thus increasing the measurement time by the factor of three for the same statistics.

There is the option to run the ETS in a half synchronized way with a trigger pulse from the ETSL. Instead of starting the system periodically by the internally generated extraction clock, the circuitry waits for the external trigger from the ETSL to get started. A jitter of approximately $26.5 \mathrm{~ns}$ relative to the external trigger will occur to get the ETS internal state machine synchronized. This synchronized mode will be used when both channels are active because otherwise cross talk between the two channels due to the fast pulsers is inevitable.

For testing the electronics, a stimulator pulse is available that generates an analogue signal from 1 to $250 \mathrm{~ns}$ width, and from 10 to $500 \mathrm{mV}$ height. Width and amplitude are 8 bit programmable. This internal calibrator generates ADC and TDC data during ground tests and in space to verify the time scale, the trigger levels and the ADC conversion. The occurrence of the pulse in the TOF test spectrum is 13 bit programmable ( $\sim 185 \mathrm{~ns}$ to $217 \mu \mathrm{s})$.

\section{Results}

Shown in Figure 20 is a mass spectrum of the calibration compound heptacosafluorotributylamine $\left.\left(\mathrm{CF}_{3}\left(\mathrm{CF}_{2}\right)_{3}\right)_{3} \mathrm{~N}\right)$ recorded with the RTOF prototype demonstrating that the mass range covered by RTOF exceeds $500 \mathrm{amu}$. The labelled peaks are fragments of the parent molecule at $671 \mathrm{amu}$, which is not observed. The abundance of the different fragment peaks agree reasonable well with the tabulated values (NIST Chemistry WebBook, http://webbook.nist.gov/chemistry/), which have been recorded with different types of mass spectrometers. For data analysis, the 


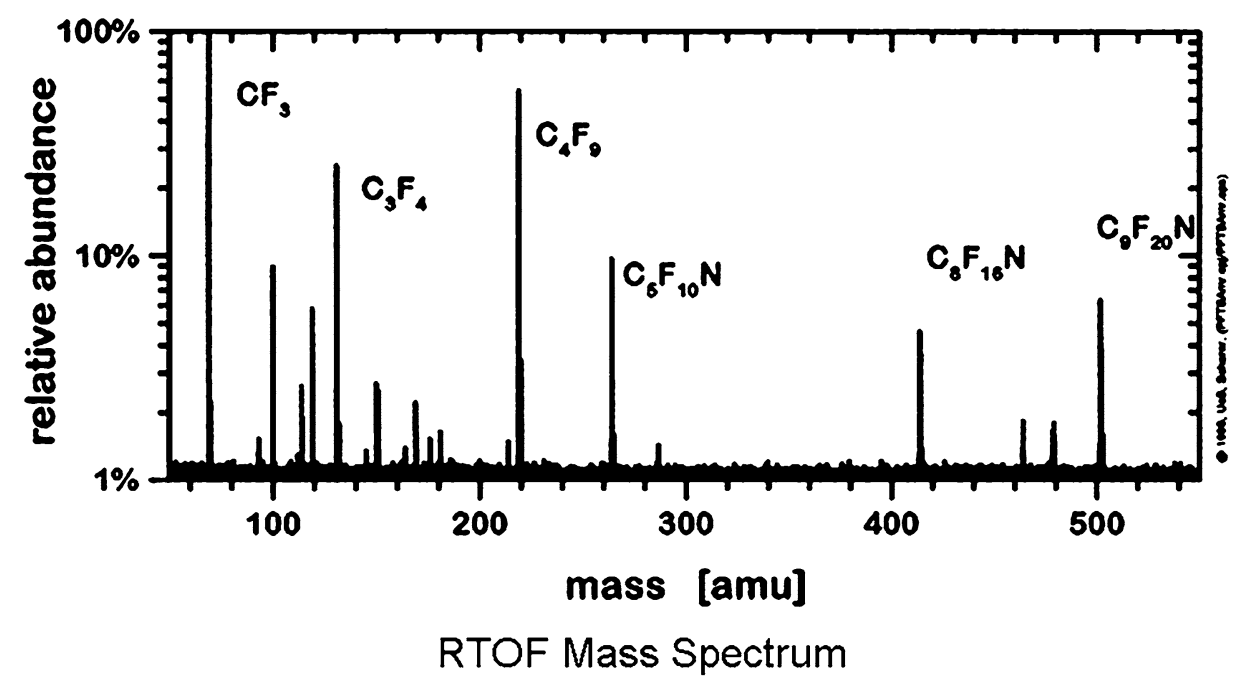

Figure 20. RTOF mass spectrum (prototype) of the calibration compound heptacosafluorotributylamine $\left.\left(\mathrm{CF}_{3}\left(\mathrm{CF}_{2}\right)_{3}\right)_{3} \mathrm{~N}\right)$.

calibrations of the fragment distribution will be done with the flight-spare model of RTOF. The mass range of RTOF is in principle unlimited. However, the limited size of the accumulation memories limits the mass range to be covered. Of course, if the start of the data taking is delayed with respect to the extraction pulse, higher masses will fall into the covered TOF window at the expense of lower mass ions. The electron impact ionization is the other limiting factor for the mass range, since this kind of ionization leads to fragmentation, which becomes dominant for large molecules and limits the mass range to about $1000 \mathrm{amu}$.

Figure 21 shows a RTOF storage source mass spectrum recorded in flight with the ETS on 22 March 2005, using the GCU. Mass peaks around $m / q=84$ are due to $\mathrm{Kr}$, at $m / q=44 \mathrm{amu}$ to $\mathrm{CO}_{2}$, and at $m / q=4 \mathrm{amu}$ to He from the GCU gas mixture. In addition, there are doubly-charged $\mathrm{Kr}$ ions around $m / q=42 \mathrm{amu}$, the fragments $\mathrm{CO}, \mathrm{O}$, and $\mathrm{C}$ from $\mathrm{CO}_{2}$, The remaining peaks are residual gas in the vicinity of the spacecraft at a total pressure of $5 \times 10^{-11}$ mbar measured with COPS. The GCU gas introduced into the storage ion source represents a pressure of about $1 \times 10^{-9}$ mbar. From the time of flight the mass per charge of any recorded ion can be calculated if the time of flights for two masses are known. Either these two masses are determined by a measurement with the in-flight calibration system or two masses can be unambiguously identified in the recorded mass spectrum. In our case we used the time of flights of mass $4(\mathrm{He})$ and of mass $44\left(\mathrm{CO}_{2}\right)$ to establish the mass scale. The choice is not critical as long as the two masses are sufficiently far apart in mass. The mass $m$ is then derived from the TOF $t$ by $m=c\left(t \times t_{0}\right)^{2}$, with $c$ and $t_{0}$ two constants derived from the calibration by some simple algebra 


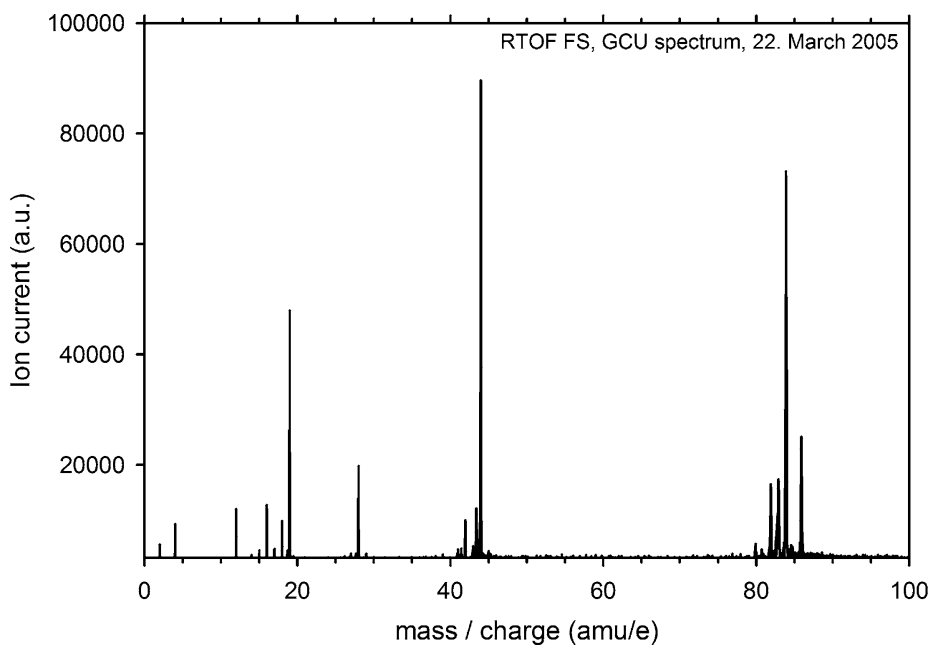

Figure 21. Mass spectrum of the in-flight calibration gas consisting of a mixture of $\mathrm{He}, \mathrm{CO}_{2}$, and $\mathrm{Kr}$ taken in space. Gas pressure in the source was $1 \times 10^{-9} \mathrm{mbar}$, integration time $200 \mathrm{~s}$.

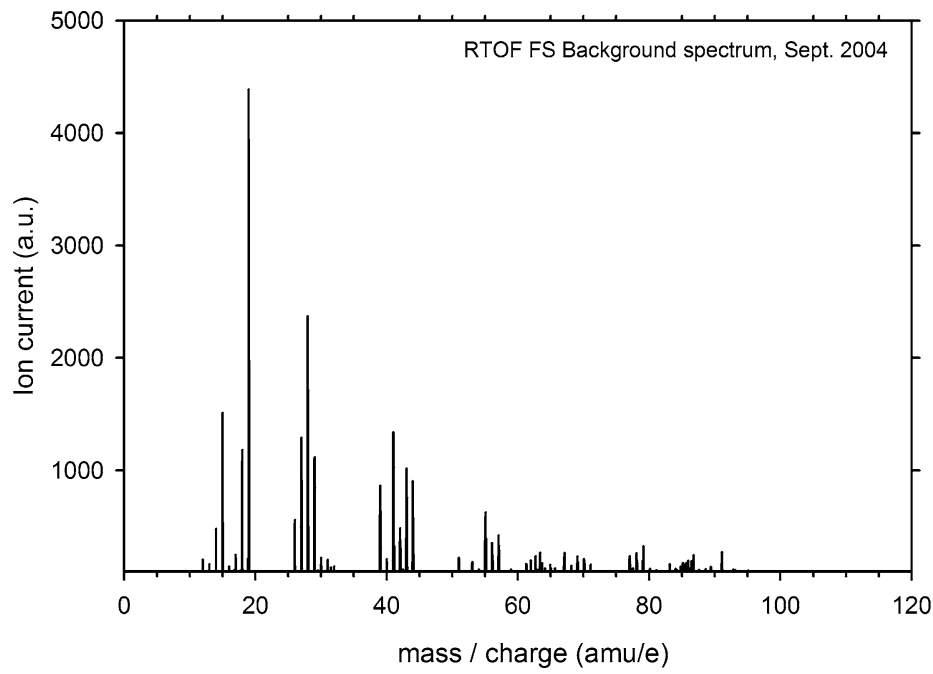

Figure 22. Background spectrum from the storage source channel recorded in space in September 2004 at an ambient pressure of $6 \times 10^{-9}$ mbar.

(e.g., Scherer et al., 2005). Using this mass scale calibration the mass of any mass line in the spectrum is known with an accuracy of about $20 \mathrm{ppm}$ (Scherer et al., 2005). Figure 22 shows a background mass spectrum from space (September 2004) taken with the storage source channel of RTOF. The ambient pressure recorded by the pressure sensor COPS was around $6 \times 10^{-11}$ mbar. 


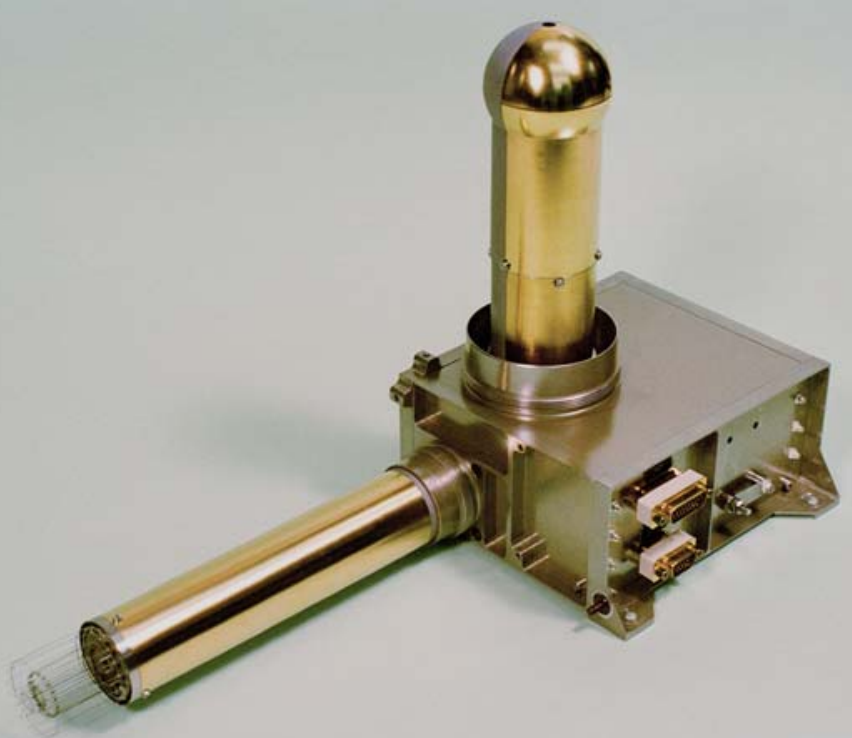

Figure 23. Picture of the ROSINA COPS-FS. The nude gauge is pointing to the left and the ram gauge is mounted on the top of the electronics housing. Connectors (with connector-savers) to the DPU are fitted into the right panel of the electronics box.

\section{COPS}

COPS consists of two sensors based on the extractor-type ionization gauge principle (Redhead, 1966). The "nude gauge" measures the total pressure (more exactly, the total neutral particle density) of the cometary gas. The "ram gauge" analyzes the ram pressure which is equivalent to the cometary gas flux. The generated ion currents are measured by corresponding high sensitivity electrometers. Depending on the measurement mode of the electrometer, COPS has a time constant of about 1-10 s. Both gauges and the electronics housing of the flight model are shown in Figure 23.

\section{The Nude Gauge}

Figure 24 shows a drawing of the COPS nude gauge. Free electrons emitted from the $17 \mathrm{~mm}$ hot filament at the potential of $+30 \mathrm{~V}$ are accelerated toward the cylindrical anode grid (22 mm diameter and $34 \mathrm{~mm}$ in height) placed at $180 \mathrm{~V}$. Ionized gas atoms and molecules are collected by the cathode hidden below the base plate. To increase the ion current yield a hemispherical reflector is mounted around the cathode, see also Figure 24. This reflector is set to a potential of $110 \mathrm{~V}$. The measured ion current is directly proportional to the particle density in the ionization volume of the nude gauge. The gauge is decoupled from the surrounding plasma by an outer grid maintained at $12 \mathrm{~V}$ compared to the spacecraft potential. For redundancy two 


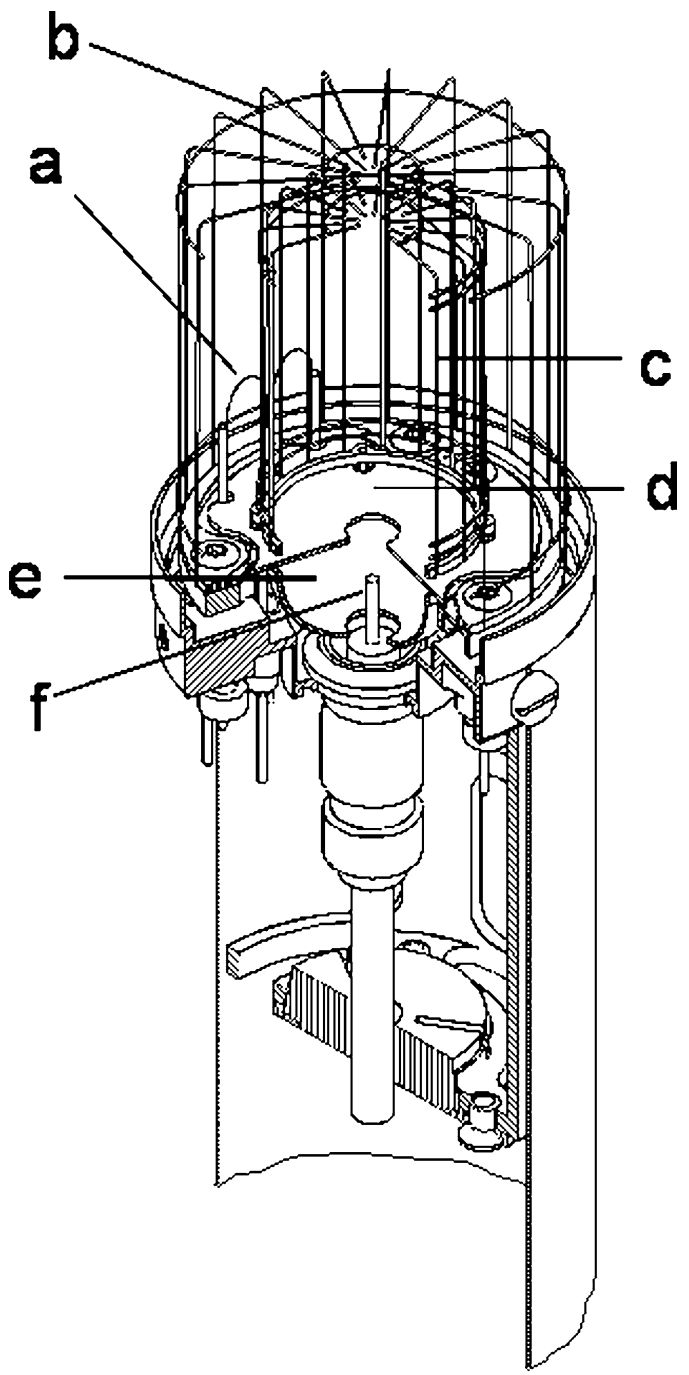

Figure 24. Drawing of the COPS nude gauge showing important components: (a) two filaments, $28 \mathrm{~V}$, (b) outer grid, $-12 \mathrm{~V}$, (c) inner grid, $180 \mathrm{~V}$, (d) base plate, $0 \mathrm{~V}$, (e) reflector, $110 \mathrm{~V}$, (f) ion collector.

filaments made from Wolfram doted with 3\% Rhenium (3 ReW) (as flown on the Giotto mission (Krankowsky et al., 1981)) are available, addressable by a switch. Each filament can emit up to $1 \mathrm{~mA}$ regulated on the current trapped by the anode grid. Taken into account the sensitivity of the electrometer and the X-ray limitation, the nude gauge can measure pressure values between $4 \times 10^{-11}$ and $10^{-5} \mathrm{mbar}$.

Laboratory calibration of the flight model at our CASYMIR facility yields a sensitivity of $20 \mathrm{mbar}^{-1}$ for nitrogen at $100 \mu \mathrm{A}$ electron emission. Additional calibration details are presented in Graf et al. (2004). 


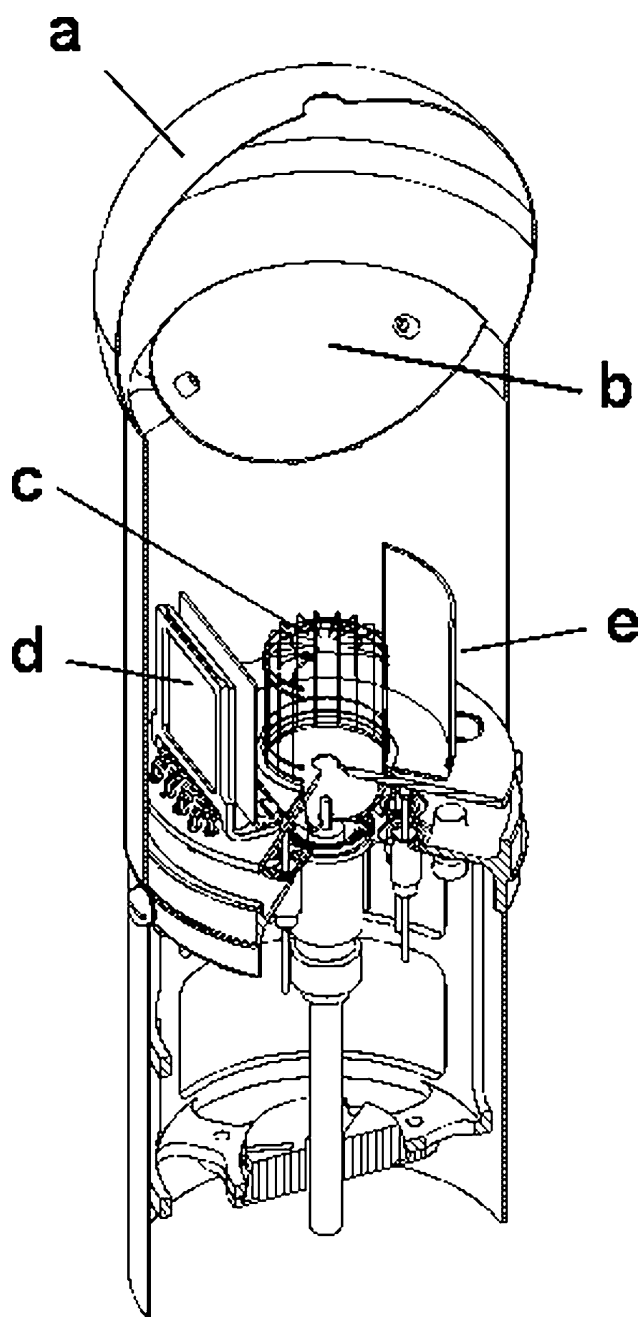

Figure 25. Drawing of the COPS ram gauge showing important components: (a) equilibrium sphere, (b) inner shield, (c) anode grid, $180 \mathrm{~V}$, (d) microtip, (e) electron repeller. The lower part with base plate, ion reflector, and collector is basically the same as for the nude gauge.

\section{The Ram Gauge}

The construction of the ram gauge is shown in Figure 25. A spherical cavity, $60 \mathrm{~mm}$ diameter with a $6 \mathrm{~mm}$ aperture facing the comet, stands on a hollow boom. A screen prevents the gas from directly impinging into the boom where the density is measured. The conductance of the top aperture is $3.41 \mathrm{~s}$ for water at $200 \mathrm{~K}$, giving an equilibrium time (Bermann, 1985) of less than $200 \mathrm{~ms}$ for the system. The real response time of the instrument is longer as it is driven by the electrometer used to measure the ion current. This configuration allows the gas to be isotropized and thermalized to the wall temperature before entering the ionization volume. 
The created ions are collected by a three-element lens-like configuration consisting of the anode grid, the base plate, and the reflector. The reflector is a hemisphere of $8 \mathrm{~mm}$ radius with an apex aperture, through which is mounted the collector $(0.15 \mathrm{~mm}$ diameter, $3 \mathrm{~mm}$ long). The anode grid $(16 \mathrm{~mm}$ diameter and $19 \mathrm{~mm}$ in height) is at $180 \mathrm{~V}$, the base plate with an aperture of $3.4 \mathrm{~mm}$ diameter at its center is at $0 \mathrm{~V}$. The nitrogen sensitivity of the flight model is $5 \mathrm{mbar}^{-1}$ for pressure values between $10^{-10}$ and $10^{-4}$ mbar, see also Graf et al. (2004).

As the wall temperature of the equilibrium sphere and the boom should be the same as the surrounding cometary coma a cold electron source has to be used for the electron impact ionization process. Therefore, the usual hot filament design was replaced by a microtip field-emitter device.

\section{The Microtips}

The microtips, of the Spindt type (Meyer, 1966; Constancias, 1998), were introduced into this type of set-up by Baptiste and Bieth (1996). The microtips have a resistive layer (Levine, 1996) that increases emission stability and serves as ballast in case of arc generation. For this type of micro-emitters a lifetime of $20,000 \mathrm{~h}$ is given by the manufacturer. Tests have been carried out to evaluate their resistance to the cometary environment, and the influence of certain gases $\left(\mathrm{O}_{2}, \mathrm{H}_{2}\right)$ (Temple, 1999).

The emitter, shown in Figure 26, is made of more than 1.8 million tips arrayed in $32 \times 32$ pixels, representing an emitting area of $14 \mathrm{~mm} \times 14 \mathrm{~mm}$. The 1024 pixels were grouped by bonding on a ceramic with gilded tracks in eight interlaced groups of vertical lines. This special arrangement gives eight independent emitters that can be addressed separately, either sequentially or jointly. Each group can deliver $1 \mathrm{~mA}$ electron emission current at $70 \mathrm{~V}$ extraction voltage as it is shown in Figure 27. Such an electron emitter is of particular interest for space applications because of its low power consumption.

\section{Mechanical/Structure/Electronics}

The nude and the ram gauge are each mounted at the end of a boom to avoid direct gas reflections from the payload platform or the nearest instruments, see also Figure 23. For mechanical stiffness, and accommodation for the launch, the booms are limited to lengths of $25 \mathrm{~cm}$. In order to preserve cleanliness, the two gauges were constantly purged with nitrogen until the launch.

The three electronic boards are housed in a $165 \mathrm{~mm} \times 140 \mathrm{~mm} \times 75 \mathrm{~mm}$ box that also supports the booms. The instrument mass is $1.5 \mathrm{~kg}$. The digital board controls the link with ROSINA's DPU. The other two boards contain the high voltage supplies for both gauges and the corresponding electrometers. Each electrometer has three ranges ( $10 \mathrm{M} \Omega, 1 \mathrm{G} \Omega$, and $100 \mathrm{G} \Omega$ with $1 \mu \mathrm{F}$ integration capacitor), switched by DPU commands. The measured value is converted by a 12 bit ADC and stored as a housekeeping value. Depending on the selected electrometer range, ion currents between $0.1 \mathrm{pA}$ and $1 \mu \mathrm{A}$ can be measured. 


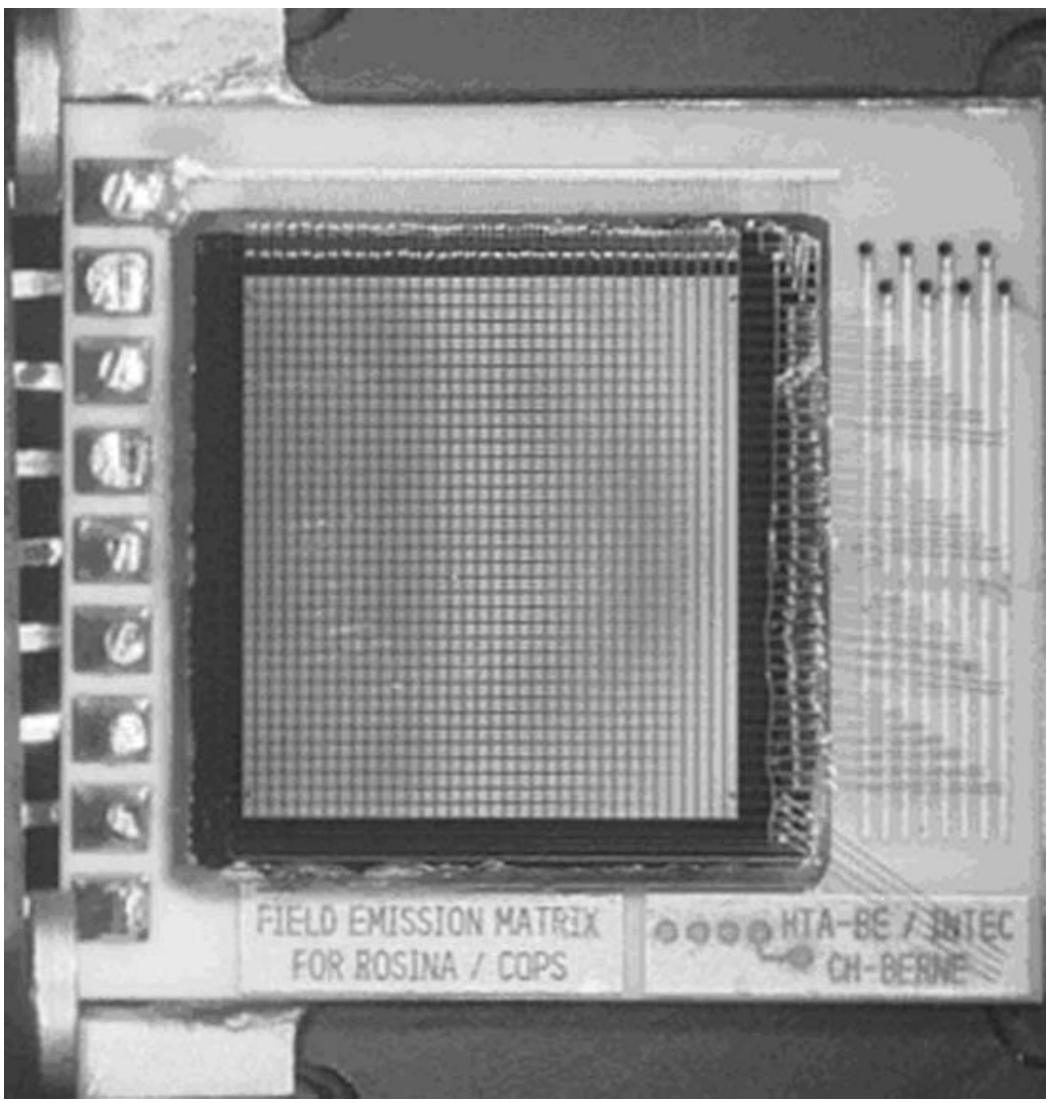

Figure 26. The COPS microtip array on its ceramic support. They are assembled in eight groups of four horizontal lines each, each line consisting of 32 pixels. The eight groups are interlaced so that, from one group to another, the emission pattern and thus the sensitivity of the gauge does not change. On the top side of the ceramic, a line provides the $110 \mathrm{~V}$ for the microtip front. The eight lines at the right enable the back potential of each group to be addressed independently. The four lines at the bottom right are not used.

Two sensors are used to measure the temperatures of the electronics and the ram gauge. The total nominal power consumption is $7 \mathrm{~W}$ at $28 \mathrm{~V}$ primary, with $2.4 \mathrm{~W}$ for the nude gauge and $0.7 \mathrm{~W}$ for the ram gauge.

The ram gauge boom points toward the comet, while the nude gauge boom is parallel to the solar panels. Half of COPS will never be exposed to the Sun, so half of each boom is sandblasted and the other half is gold-plated (see also Figure 23). On the spacecraft, the electronics box is protected by a multi-layer insulation.

\section{Results}

Both gauges were successfully switched on and tested during a 2 days commissioning phase in March 2004. For the nude gauge modes with up to $200 \mu \mathrm{A}$ electron emission were used for the left and the right filament. Group one to three of the 


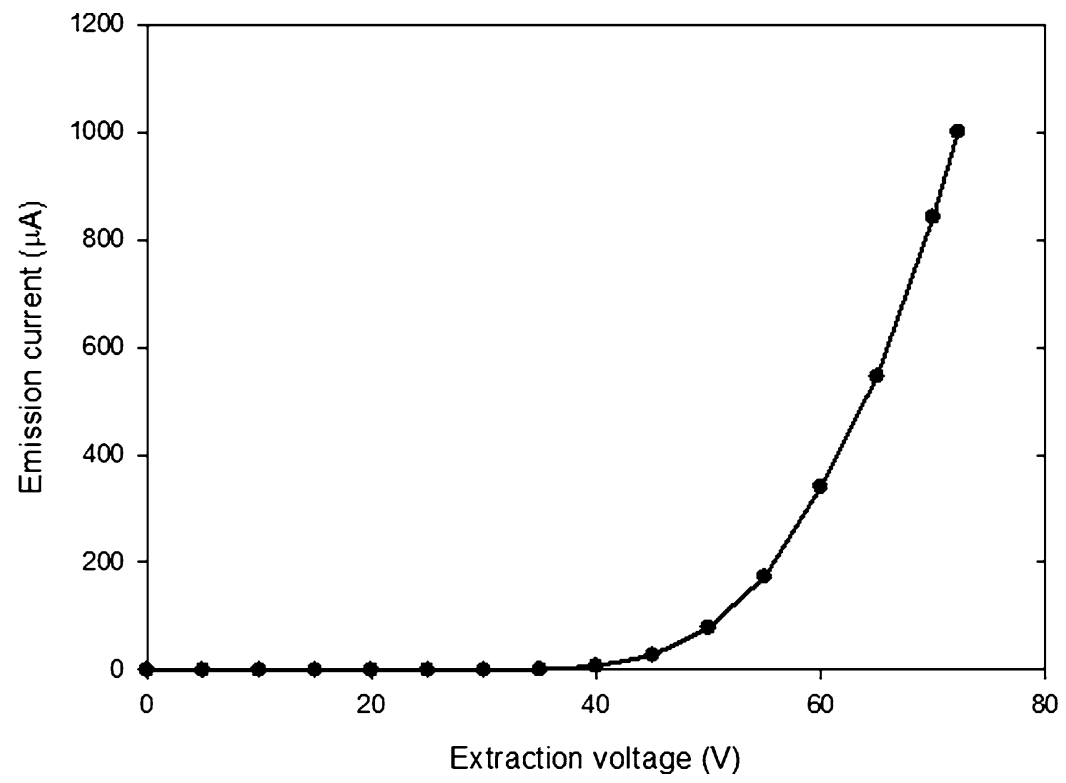

Figure 27. Typical electron emission curve obtained with one of the eight groups of the microtip array.

ram gauge microtip were conditioned with up to $18 \mu \mathrm{A}$ electron emission, and the remaining groups, four to eight, were tested with up to $5 \mu \mathrm{A}$. The very strict time constraints during the commissioning did not allow for further conditioning of the microtip array at higher electron emissions.

An overview over several pressure measurements during one and half year after the launch is given in Figure 28. Over the entire time range a pressure decrease is visible. This is due to a diminishing outgassing of the instrument and spacecraft surfaces.

Most of these measurements were recorded in the COPS monitoring mode. In this mode, the pressure value is reported as a housekeeping value every minute. The actual pressure values are polled by the DPU every two second and a running average over five data points is calculated.

\section{Digital Processing Unit (DPU)}

\section{DPU Hardware Design}

A picture of the DPU is given in Figure 29. Principal drivers of the DPU design (Figure 30) are

- optimum use of the allocated telemetry rate,

- single-failure tolerance for all functions serving more than one sensor,

- independence of availability of radiation hardened parts. 


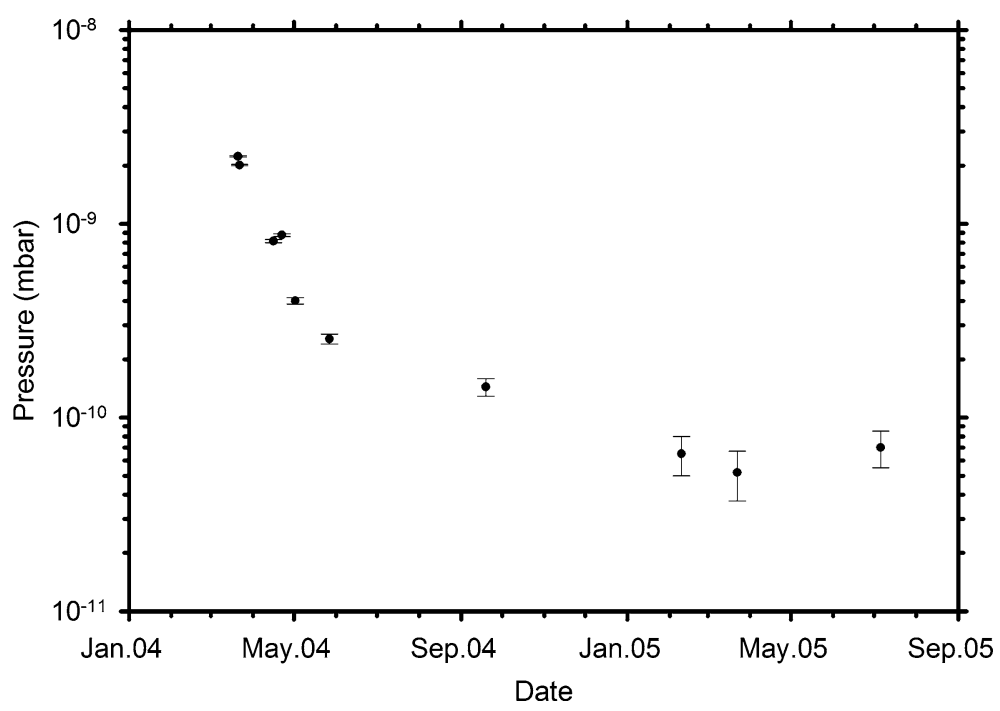

Figure 28. Overview of the measured COPS pressure values onboard the Rosetta spacecraft as a function of the time in UTC. The first measurements were taken 18 days after launch. Because of the diminishing outgassing of the spacecraft lower final pressure values can be measured with increasing mission time.

The primary data rate of all three detectors exceeds the maximum spacecraft telemetry rate $\left(20 \mathrm{kbit} \mathrm{s}^{-1}\right)$ by more than three orders of magnitude. Reducing the amount of scientific data is therefore a fundamental need. It is achieved at two levels: hardware-based integration within the sensor electronics, and subsequent S/W processing by

- spectrum windowing,

- averaging, resulting in degraded mass and/or time resolution,

- lossless compression (modified Rice PSI14 (Rice, 1991)), and task specific lossy compression.

All $\mathrm{S} / \mathrm{W}$ processing is performed in the DPU by a 32-bit digital signal processor (DSP, TSC21020F) with a large and fast SRAM memory (3 MB program, $8 \mathrm{MB}$ data memory).

All DPU functions are duplicated and organized into two independent (cold redundant) branches except the three-sensor interfaces and the hard core for selection of the active branch. Cross-strapping is applied between each sensor interface and each DPU branch, and between each DPU power converter and each DPU branch.

The program and data memory are $\mathrm{H} / \mathrm{W}$-protected against single event upsets (SEUs) and permanent device failures. For adaptation to 8-bit-wide memory devices a "Single 8-bit Symbol Error Correction, Double 8-bit Symbol Error Detection" $(48,72)$-Reed-Solomon Code (Reed and Solomon, 1960) is used. Periodic scrubbing of the memories prevents the accumulation of non-correctable double symbol 


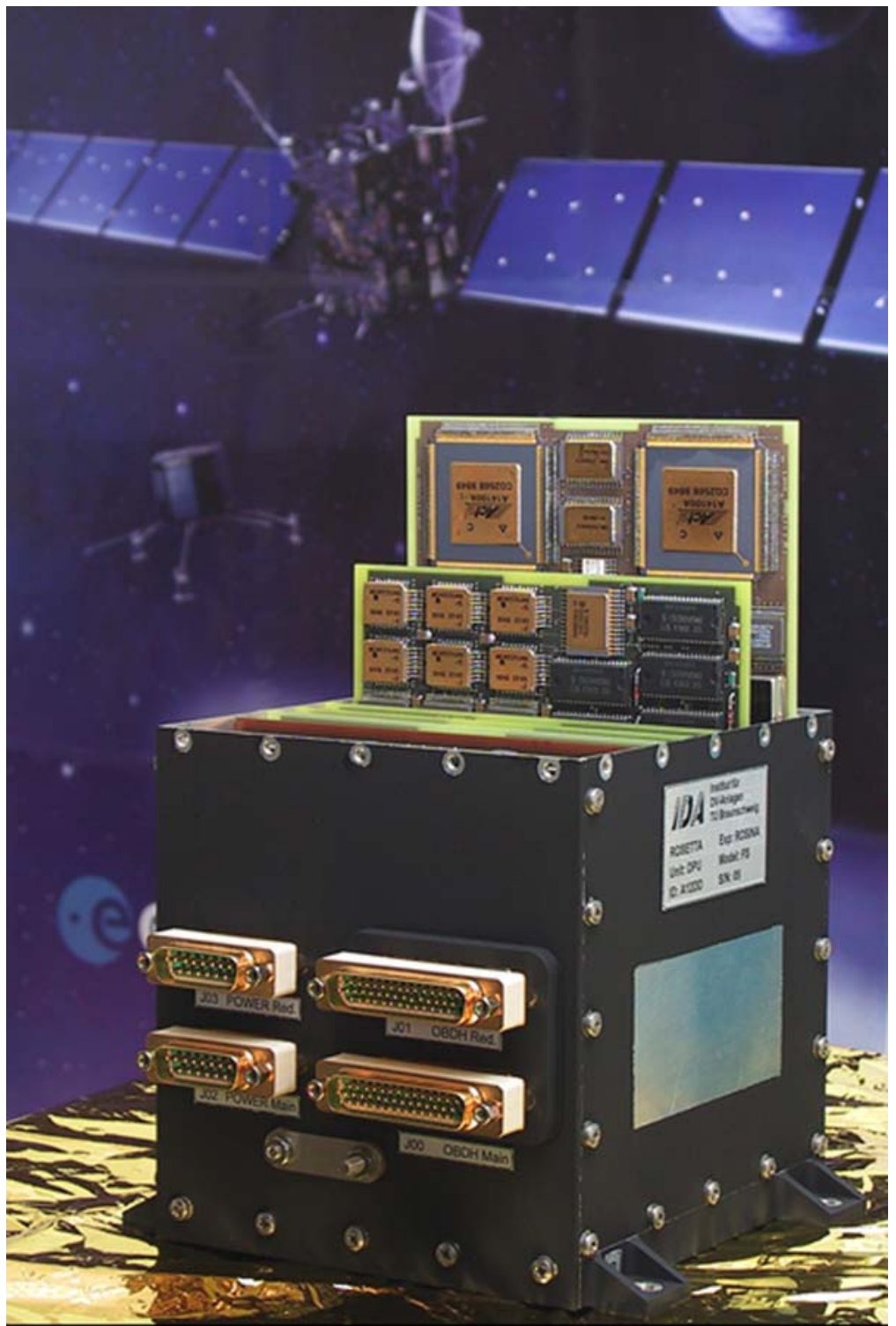

Figure 29. Picture of the ROSINA DPU.

errors. The remaining SEU-induced undetected errors ( $>2$-symbol memory errors) can cause a breakdown in program execution, which is monitored by a watchdog circuit.

Single event latch-up (SEL) induced overcurrents are detected by current monitoring. Each of both DPU branches is protected by nine current monitors. If an overcurrent occurs, the DPU branch is de-energized by a fast $(\mu \mathrm{s})$ current breaker. 


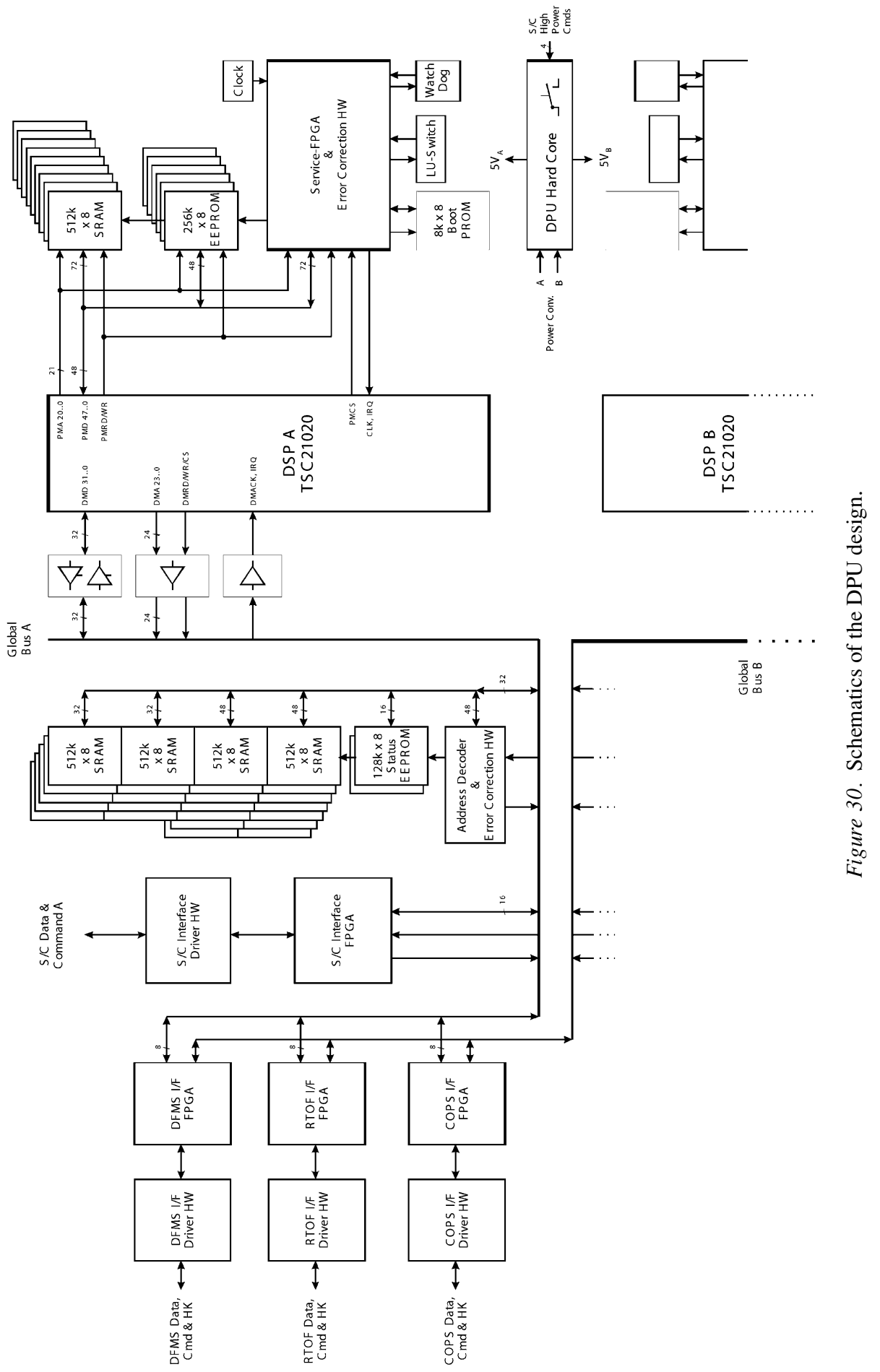




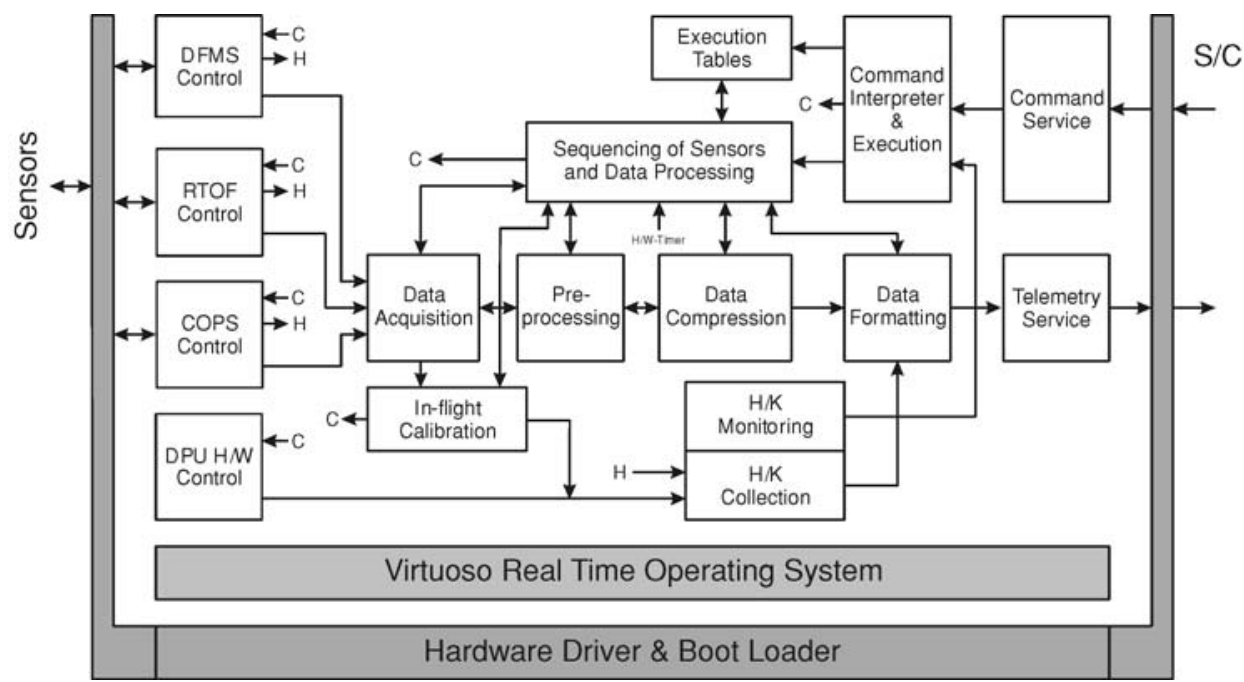

Figure 31. Schematics of the DPU layer model.

\section{DPU Software Design}

The DPU S/W is based on the real-time multitasking operating system Virtuoso (Eonic Systems) that provides:

- preemptive, event-driven scheduling,

- dynamically prioritized tasks,

- synchronization and communication facilities (semaphores, mailboxes, queues, timers),

- dynamic memory management, and

- handling of multilevel device interrupts.

All S/W tasks are grouped in a layer model with five layers (Figure 31):

(5) Scientific Software,

(4) Operation Control (command execution, emergency mode, In-flight calibration, etc.),

(3S) Service Functions (command interpreter, housekeeping collection, data compression, etc.),

(3) Element Functions (detector on/off, data acquisition/handling, etc.),

(2) Subelement Functions (direct control of subelements), and

(1) Low Level S/W (H/W driver, I/O control).

\section{Electrical Ground Support Equipment (EGSE)}

The same test equipment will be used during all program phases: unit level electrical tests, system level electrical tests, and flight operations. The EGSE hardware 


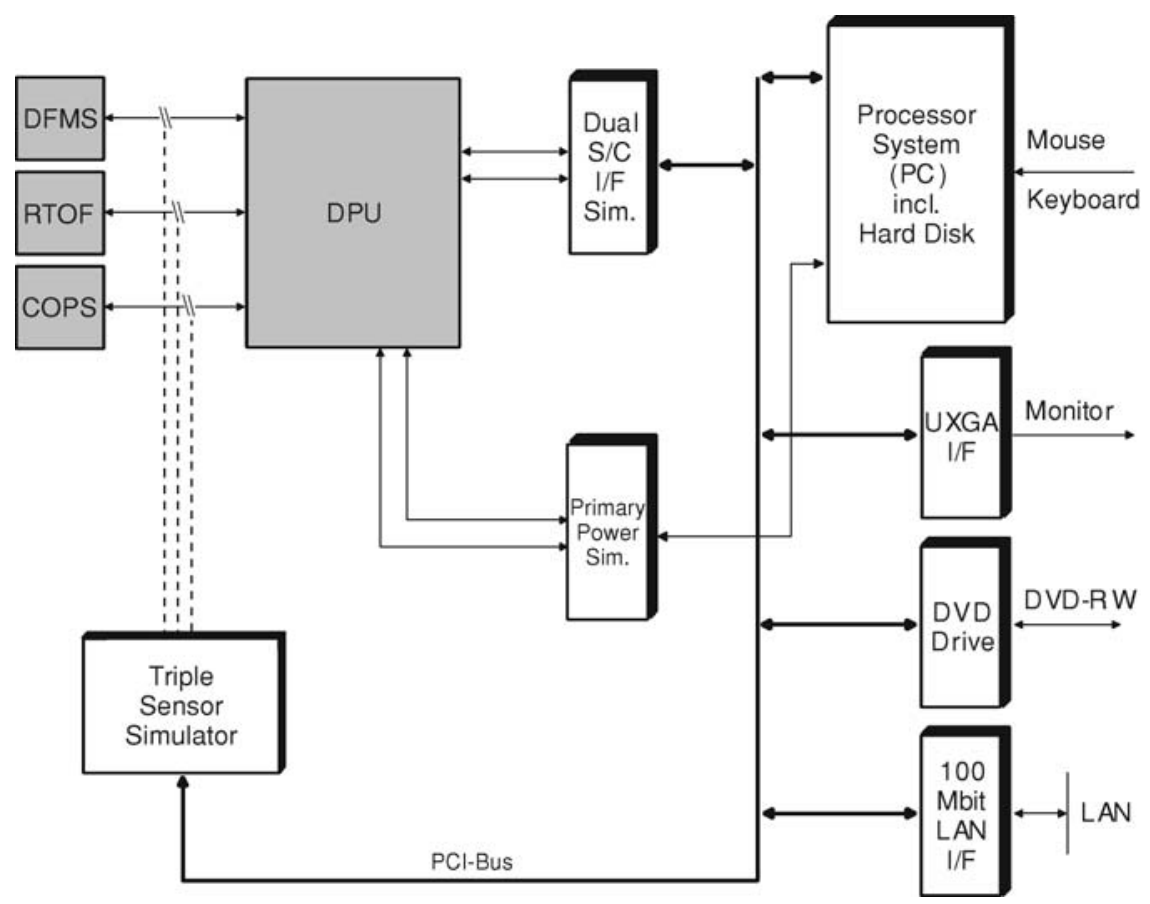

Figure 32. Schematics of the electrical ground support equipment EGSE.

architecture is based on a modular design (Figure 32). The different subsystems are connected via a standard internal bus (32-bit PCI). The main components are:

- a Microprocessor System (Pentium II PC for commanding, data decoding, data display, etc.),

- a dual S/C OBDH interface simulator,

- three sensor electronics simulators, and

- a primary power supply unit.

External PCs can be connected to the EGSE via a private network (Ethernet) for remote data analysis and instrument control.

The PC operating system "Ground Support Equipment Operating System" (GSEOS 5.0) running with Windows NT consists of the following packages:

- $\mathrm{I} / \mathrm{F}$ drivers,

- Block Data Manager (BDM) for central data handling,

- command processor (control of both the instrument hardware and the $\mathrm{I} / \mathrm{F}$ simulator),

- quick-look data display for displaying of instrument telemetry data and I/F simulator status, 
- decoder (monitoring and user defined mathematical expressions on data items),

- network for connection to several remote PCs and to CCS/Ground Segment, and

- recorder for recording of real-time telemetry data, I/F simulator status data and commands.

\section{Scientific Closure}

Table IV shows the scientific objectives that will be addressed using the data products from the ROSINA investigation. In addition to ROSINA's specific science objectives listed in the table, the data products will provide key information for additional science objectives of other Rosetta Orbiter and Lander instruments. Collaboration between ROSINA and other Orbiter and Lander investigations will greatly enhance the scientific results in several key areas including: dust-gas interaction, gas-plasma interaction, causes of cometary activity, and compositional differences within the nucleus.

A complete understanding of the dust-gas interaction will require collaboration between ROSINA and the dust investigation. The comet produces approximately equal concentrations of gas and dust (Balsiger et al., 1988) and there is a strong indication that this combination is responsible for extended sources such as $\mathrm{CO}$ in comet Halley (e.g., Eberhardt, 1999). Extended observations of the comet by both ROSINA and the dust experiment will be exploited in a search for other extended

TABLE IV

ROSINA sensors, data products and science objectives

\begin{tabular}{|c|c|c|}
\hline Sensor & Data product & Science objective \\
\hline \multirow[t]{6}{*}{ DFMS/RTOF } & $\begin{array}{l}\text { High resolution and high } \\
\text { sensitivity mass spectra }\end{array}$ & Origins of comets \\
\hline & & $\begin{array}{l}\text { Origins of organic material in } \\
\text { comets }\end{array}$ \\
\hline & $\begin{array}{l}\text { Heliocentric/temporal } \\
\text { dependence }\end{array}$ & $\begin{array}{l}\text { Onset of cometary activity, } \\
\text { composition changes in the } \\
\text { coma }\end{array}$ \\
\hline & Cometocentric dependence & $\begin{array}{l}\text { Coma chemistry, gas-dust } \\
\text { interaction }\end{array}$ \\
\hline & & Causes of cometary activity, \\
\hline & $\begin{array}{l}\text { Detailed mapping of active and } \\
\text { quiescent regions }\end{array}$ & $\begin{array}{l}\text { Composition of the nucleus } \\
\text { compositional differences } \\
\text { within the nucleus }\end{array}$ \\
\hline COPS & $\begin{array}{l}\text { Neutral pressures, velocities, } \\
\text { temperatures }\end{array}$ & Coma gas-dust dynamics \\
\hline
\end{tabular}


gas sources and a complete characterization of the known extended sources and their origin within the dusty atmosphere.

Similarly, an understanding of the gas-plasma interaction will require collaboration between ROSINA and the plasma experiment. Basic quantities such as the gas production rate of the comet obtained from ROSINA will be important elements in the understanding of the plasma observations. Likewise, the plasma flow velocity, electron temperature and magnetic field will be important quantities for determining and checking the location of the contact surface near the comet when it is close to the Sun. Low-energy ion flow inside the contact surface is significantly affected by the presence of this barrier and its location will be important in interpreting the ROSINA ion observations.

A complete understanding of the causes of cometary activity and compositional differences within the nucleus will require collaboration between ROSINA and several Orbiter and Lander investigations. One important aspect to be investigated is the composition of volatiles measured by ROSINA and the composition of nonvolatile surface components measured by the Lander. A cross-check of the relative composition of these two cometary components is required to account completely for cometary composition and to understand how (or if) the cometary coma differs from the evacuated material in the mantle. This combination of Orbiter and Lander composition measurements will be key in resolving the question of the ultimate fate of comets in the solar system.

Causes of cometary activity and compositional differences within the nucleus will also be investigated through collaboration with other Orbiter investigations. One important collaboration will be the coordinated mapping of cometary active regions by ROSINA, the camera and the dust investigations. Possible compositional differences of the active regions will be measured directly with the narrow field of view part of ROSINA's DFMS. In coordination with camera and dust observations, these regions will be localized and identified. Possible compositional differences of each of these regions will be investigated periodically during the mission to determine if gas from these regions changes with increasing cometary activity.

\section{Acknowledgments}

The authors would like to thank the following institutions and agencies, which supported this work: Work at UoB was funded by the State of Bern, the Swiss National Science Foundation, and by the European Space Agency PRODEX program. Work at CETP was financed by CNES through grant ROSETTA 98CNES7083. The work at CESR was funded by a CNES grant. The work at MPS, Lindau was funded by the Max-Planck-Gesellschaft zur Förderung der Wissenschaften and the DLR through grant 50 QP 97040. The authors from Belgium like to thank the Belgian Federal Office for Scientific, Technical and Cultural Affairs (DWTC-SSTC) for approval of the ROSINA space project. This work was supported by means of three 
contracts granted to BIRA-IASB, OIP and IMEC by the European Space Agency PRODEX Program (ESA PRODEX Experiment Arrangement no. 90020). The US participation in the ROSINA project is funded by NASA through the Jet Propulsion Laboratory under contract 1202479 . We herewith acknowledge gratefully the work of numerous engineers from the institutions involved in ROSINA who contributed significantly to the ROSINA instrument. Our special thanks go to the two referees who read the manuscript very thoroughly and helped to improve it.

\section{References}

Balsiger, H., et al.: 1988, Scientific American, 96.

Baptist, R., and Bieth, C.: 1996, J. Vac. Sci. Tecnol. B 14(3), 2119.

Bermann, A.: 1985, Total Pressure Measurements in Vacuum Technology, Academic Press, San Deigo, Chapter 8, p. 355.

Berthelier, J. J., Illiano, J. M., Nevejans, D., Neefs, E., Arijs, E., and Schoon, N.: 2002, Int. J. Mass Spectr. 215(1-3), 89.

Constancias, C.: 1998, PhD thesis, Université de Grenoble, Saint Martin d'Heres, France.

Eberhardt, P.: 1999, Space Sci. Rev. 90, 45.

Graf, S., Altwegg, K., Balsiger, H., Jäckel, A., Kopp, E., Langer, U., Luithardt, W., Westermann, C., and Wurz, P.: 2004, J. Geophys. Res. 109, E07S08, doi: 10.1029/2003JE002188.

Hohl, M., Wurz, P., Scherer, S., Altwegg, K., and Balsiger, H.: 1999, Int. J. Mass Spectr. 188, 189.

Kissel, J., et al.: 1986, Nature 321, 336.

Krankowsky, D., et al.: 1981, Scientific and Experimental Aspects of the Giotto Mission, 3-7 (ESA SP-169, 1981), p. 127.

Irvine, W.: 1999, Space Sci Rev. 99(1-2), 203.

Levine, J. D.: 1996, J. Vac. Sci. Technol. B 14(3), 2008.

Mamyrin, B. A., Karataev, V. I., Shmikk, D. V., and Zagulin, V. A.: 1973, Sov. Phys. JETP 37(1), 45.

Mattauch, J., and Herzog, R.: 1934, Z. Physik 89, 786-795.

Matsuda, H., and Fujita, Y.: 1975, Int. J. Mass Spectr. 16, 395.

Meyer, R.: 1966, Le vide, 282, 478.

Nevejans, D., Neefs, E., Kavadias, S., Merken, P., Van Hoof, C., Gramegna, G., Bastiaens, J., and Dierickx, B.: 2000, Rev. Sci. Instrum. 71(11), 4300.

Nevejans, D., Neefs, E., Kavadias, S., Merken, P., and Van Hoof, C.: 2002, Int. J. Mass Spectr. 215(1-3), 77.

Redhead, P. A.: 1966, J. Vacuum Sci. Tecnol. 13, 173.

Reed, I., and Solomon, G.: 1960, J. Soc. Ind. Appl. Math. [SIAM J.] 8, 300-304.

Rice, R. F. : 1991, JPL Publication 91-3, Jet Propulsion Laboratories, November 1991.

Shelley, E. G., et al.: 1995, Space Sci. Rev. 71(1-4), 497.

Siegmund, O. H. W., Kromer, K. E., Wurz, P., Schletti, R., and Cottard, H.: 2000, Proc. SPIE Int. Soc. Opt. Eng. 4140, 229.

Scherer, S., et al.: 2005, Int. J. Mass Spectr., in press.

Schletti, R., Wurz, P., Scherer, S., and Siegmund, O. H.: 2001, Rev. Sci. Instr. 72, 3.

Temple, D.: 1999, Mater. Sci. Eng. R24, 185.

Westermann, C., Luithardt, W., Kopp, E., Koch, T., Liniger, R., Hofstetter, H., Fischer, J., Altwegg, K., and Balsiger, H.: 2001, Meas. Sci. Technol. 12(9), 1594.

Wiley, W. C., and McLaren, I. H.: 1995, Rev. Sci. Instr. 26(12), 1150.

Wurz, P., and Gubler, L.: 1994, Rev. Sci. Instr. 65, 871.

Wurz, P., and Gubler, L.: 1996, Rev. Sci. Instr. 67, 1790. 